\title{
Semigroup Commutators under Differences
}

N. Th. Varopoulos

\section{Introduction}

This is the first paper on a subject whose motivation lies in a geometric problem that I shall now explain.

Motivation. Let us consider locally in $\Omega \subset \mathbb{R}^{n}$ (or in a more general $C^{\infty}$ manifold) a 'self-adjoined second order differential operator with positive characteristic ( $c f$. [13] [7] [8] [11] [10]):

$$
L f=h^{-1} \sum_{i, j}\left(\partial_{i} a_{i j} \partial_{j} h f\right), \quad f \in C_{0}^{\infty},
$$

with $h, h^{-1}, a_{i j} \in C^{\infty}$ and real and with the matrix $A=\left(a_{i j}\right) \geqslant 0$ non-negative. We associate then to $L$ a canonical distance by the following «recipe»: For every $x \in \Omega$ we say that $y \in B_{r}(x)$ lies in the $r$-ball $(r>0)$ centered at $x$ if $y$ is the end point of an absolutely continuous path $l(t)(0 \leqslant t \leqslant r)$ with $l(0)=x$, $l(r)=y$ that is «sub-unit» for $L$. This means that the speed vector

$$
i(t)=d l\left(\frac{\partial}{\partial t}\right)=\xi
$$

is almost everywhere subunit for $L$, i.e. that

$$
\left(\sum \xi_{i} \lambda_{i}\right)^{2} \leqslant \sum a_{i j} \lambda_{i} \lambda_{j}, \quad \lambda_{i} \in \mathbb{R} .
$$

The value $d(x, y)=+\infty$ is admitted here if $y$ lies in no ball centered at $x$. 
The above operator is formally self-adjoint in $X=L^{2}\left(h^{2} d x\right)$, it can therefore be closed in $X$ and the complex powers $L^{\alpha}(\alpha \in \mathbb{C})$ of $L$ can be defined. We shall say that $L$ is subelliptic if for some positive $\epsilon>0$ we have

$$
\left\|\Delta^{\epsilon} f\right\|_{L^{2}} \leqslant C\left(\|L f\|_{L^{2}}+\|f\|_{L^{2}}\right)
$$

where $\Delta$ is of course the positive Laplacian. (Alternatively $L$ is subelliptic if the distance $d(\bullet, \bullet)$ induced by $L$ is Hölder continuous with respect to Euclidean distance ( $c f$. [11] [12]).)

Let now $L_{1}$ and $L_{2}$ be two subelliptic operators as above and let $d_{1}, d_{2}$ denote their two corresponding distances. For two fixed $\alpha, \beta>0$ (it will have to be $\beta \leqslant \alpha$ ) let us consider the following three conditions:

(i) There exists $C>0$ such that

$$
d_{1}^{\alpha}(x, y) \leqslant C d_{2}^{\beta}(x, y), \quad x, y \in \Omega .
$$

(ii) There exists $C>0$ such that

$$
\left\|L_{2}^{\beta} f\right\|_{L^{2}} \leqslant C\left(\left\|L_{1}^{\alpha} f\right\|_{L^{2}}+\|f\|_{L^{2}}\right), \quad f \in C_{0}^{\infty} .
$$

(iii) For every $1<p<+\infty$ there exists $C>0$ such that

$$
\left\|L_{2}^{\beta} f\right\|_{L^{p}} \leqslant C\left(\left\|L_{1}^{\alpha} f\right\|_{L^{p}}+\|f\|_{L^{p}}\right), \quad f \in C_{0}^{\infty} .
$$

As we shall see below it is reasonable to conjecture that the above three conditions are all equivalent.

The basic original approach to this conjecture is due to Hörmander [14] who (among other things) proved that essentially (i.e. with a loss of an $\epsilon$ !) (i) implies (ii) with $L_{2}=\Delta$ (the standard Laplacian) and

$$
L_{1}=\sum X_{j}^{*} X_{j}
$$

a «sum of squares» operators (sometimes called a «Hörmander» operator) where the $X_{j}^{\prime}$ 's are first order differential operators, without constant term, i.e. $X_{j}$ are $C^{\infty}$ fields and $X_{j}^{*}$ denotes their formal adjoints (with respect to Lebesgue measure). The equivalence of (i) and (ii) was proved in [11] for $0<\alpha, \beta \leqslant 1$ when $L_{2}=\Delta$. The implication (i) implies (ii) for arbitrary $L_{1}, L_{2}$ was shown in [15], but for $0<\alpha, \beta<1 / 2$. The equivalence of (i), (ii) and (iii) was shown in [8] for arbitrary $L_{1}, L_{2}$ but $\alpha=\beta$. Finally the implication (i) implies (iii) for $L_{2}=\Delta$ and arbitrary $\alpha, \beta>0$ was shown in [3]. In this paper the conjecture will be reinforced further and the following theorem will be proved

Theorem. Let $L_{1}, L_{2}, d_{1}, d_{2}$ be two differential operators with their corresponding distances as above and let us assume both $L_{1}, L_{2}$ are subelliptic Hör- 
mander operators. I shall further impose on $L_{1}$ the following technical condition

$$
d_{1}(x, y) \leqslant C|x-y|^{1 / 2}, \quad x, y \in \Omega,
$$

for some fixed $C>0$.

Then (i) implies (iii) for arbitrary $\alpha, \beta>0$. Conversely, if (ii) holds for some fixed $\alpha_{0}, \beta_{0}>0$ then (i) holds for $\alpha=\alpha_{0}$ and $\beta=\beta_{0}-\epsilon$ and arbitrary $\epsilon>0$.

At this stage I do not see how to eliminate the technical condition of the $1 / 2$-Hölder continuity of $d_{1}$, except by replacing it by other conditions that are equally unnatural ( $c f$. Section 8 ). On the other hand, in a second instalment of this paper I do hope to eliminate the other (artificial) condition of this theorem that $L_{1}$ and $L_{2}$ are «sum of squares». The $\epsilon>0$ in the second part of this theorem should not of course be there. At the end of this paper however, I shall give an example of two operators as above that are sums of squares for which we have: $d_{2}(\cdot, \bullet) \leqslant C_{\epsilon} d_{1}^{1-\epsilon}(\cdot, \bullet)$ holds for all $\epsilon>0$ but not for $\epsilon=0$. A phenomenon that cannot occur when $L_{1}=\Delta$ and $L_{2}$ is a sum of squares.

\section{The Commutators}

The technical result that makes everything work is a theorem on commutators of pseudo-differentials that I shall now describe in «general terms». Precise statements will be given later. Let $H_{\alpha}=\left\{f: \Lambda^{\alpha} f \in L^{2}\right\}$ with

$$
\Lambda=\left(I-\sum \frac{\partial^{2}}{\partial x_{i}^{2}}\right)^{1 / 2}
$$

denote the standard Sobolev spaces and let $L \in O P S_{1,0}^{2}$ be a pseudo-differential with a positive symbol that induces a non-negative Hilbert space operator $A$ (e.g. $A=L(x, D)+L^{*}(x, D)+c I$ or $L^{\omega}(x, D)+c I$ or simply in our special case

$$
A=\sum X_{j}^{*} X_{j},
$$

a sum of squares operator). The complex powers $A^{\sigma}(\sigma \in \mathbb{C})$ can then be defined and the basic commutation result that we shall prove in this paper is this:

For any choice $S_{j} \in O P S_{1,0}^{n_{j}}, n_{j} \in \mathbb{R}, 1 \leqslant j \leqslant k$ and any $m \in \mathbb{R}, \sigma \in \mathbb{C}$ we have (0.2) $\left\|\left[\cdots\left[\left[A^{\sigma}, S_{1}\right], S_{2}\right] \cdots S_{k}\right] f\right\|_{m} \leqslant C\left\|A^{\sigma-k / 2} f\right\|_{m+m_{1}+\cdots+n_{k}}, \quad f \in C_{0}^{\infty}$ 
$[A, B]=A B-B A$ denotes as usual the commutator of two operators and $\|\bullet\|_{\alpha}$ denote the Sobolev norms in $H_{\alpha}$. The above will be proved for sum of squares operators in this paper and we will present a general proof in a later work.

We shall see in Section 8 the incidence that this commutator theorem has on our previous geometric problem. The result $(0.2)$ has however another important consequence that I shall not develop here.

Indeed we shall see in Section 3 that for $\operatorname{Re} \sigma \geqslant 0$ we have

$$
\left\|A^{\sigma} f\right\|_{m} \leqslant C\|f\|_{m+2 \operatorname{Re} \sigma}, \quad f \in C_{0}^{\infty} .
$$

and also that for $\operatorname{Re} \sigma \leqslant 0$ we have

$$
\left\|A^{\sigma} f\right\|_{m} \leqslant C\|f\|_{m+2(1-\delta) \operatorname{Re} \sigma}, \quad f \in C_{0}^{\infty}
$$

provided that $A$ satisfies the subellipticity condition

$$
\|f\|_{1-\delta} \leqslant C\left\|A^{1 / 2} f\right\|_{L^{2}}
$$

for some $0 \leqslant \delta<1$. If we combine (0.2), (0.3), (0.4) we see that for $S_{j} \in S_{1,0}^{1}$ and $\operatorname{Re} \sigma \leqslant 0$ we have

$$
\left\|\left[\cdots\left[A^{\sigma}, S_{1}\right], \ldots, S_{k}\right] f\right\|_{m} \leqslant C\|f\|_{m+k \delta+2 \operatorname{Re} \sigma(1-\delta)} .
$$

If we combine this with Beals' characterization of pseudo-differentials ( $c f$. [16], [17], [18]) we immediately see that the subellipticity condition (0.5) for $0 \leqslant \delta \leqslant 1 / 2$ implies that $A^{\sigma} \in O P S_{\nu, \nu}^{2 \operatorname{Re}(1-\delta)}(\operatorname{Re} \sigma \leqslant 0, \delta \leqslant \nu \leqslant 1-\delta)$. This is already, of course, a theorem of Beals ( $c f$. [16]). The present method, however, allows us to handle more general operators than in [16]. In particular the pseudo-differentials do not have to be polyhomogeneous. Another advantage of this approach is that we get (for free, so to speak) all the $L^{p}$ norms. So that in (0.2), (0.3) and (0.4) we can, for operators as in (0.1), replace the $H_{\alpha}$ norms by the $L^{p}$-Sobolev norms (i.e. $\Lambda^{\alpha} f \in L^{p}$ ) and this is quite significant since, as is well-known, the general Hörmander classes $O P S_{\delta, \delta}^{0}$ do not operate on $L^{p}$ (cf. [18], [19]). At any rate, I shall say no more about that aspect of the theory here. It will be developed in a later publication.

In our commutator result (0.2) and in some of its consequences the self adjointness of the operators (Alternatively real symbols) is not essential. This can easily be seen from the proofs. I shall only consider self-adjoint operators here however because this is all that will be needed for the Geometric Applications. Concerning self-adjointness, it turs out to be irrelevant with respect to which particular measure $d \mu=h^{2} d x\left(h \in C^{\infty}\right)$ the differential operators are self-adjoint. For simplicity therefore I shall always take $h \equiv 1$ in $(0.1)$. 


\section{The Real Interpolation}

Let $X$ be a Hilbert space (more general Banach spaces can also be considered) and let $A=I+A_{1}$ where $\left(A_{1} f, f\right) \geqslant 0$ is a positive closed self-adjoint operator that generates the semigroup $e^{-t A} \in \mathscr{L}(X)$ with $\left\|e^{-t A}\right\| \leqslant e^{-t}$. I shall denote by

$$
X_{\alpha}=\left\{f \in X: A^{\alpha / 2} f \in X\right\}, \quad \alpha \in \mathbb{R} .
$$

The corresponding scale of «Sobolev spaces».

Typically $X=L^{2}\left(\mathbb{R}^{n}\right)(n \geqslant 1)$ and $A_{1}=a(x, D)+a^{*}(x, D)+c I$ for an appropriate $c>0$ where the symbol $a(x, \xi) \in S_{1,0}^{2}$ is non-negative $a(x, \xi) \geqslant 0$. More general manifolds can also be considered. Weyl calculus should of course be used here and we should set $A_{1}=a^{w}(x, D)+c I$. We shall however not do so, at least not systematically, in this paper. The fact that $\left\|e^{-t A}\right\| \leqslant e^{-t}$ implies that

$$
A^{-\nu}=c \int_{0}^{\infty} t^{\nu-1} e^{-t A} d t
$$

is a bounded operator on $X_{\alpha}(\alpha \in \mathbb{R})$ for every $\nu \in \mathbb{C}$ with $\operatorname{Re} \nu>0$. This means that the Sobolev spaces are naturally nested: $X_{\alpha} \subseteq X_{\beta}$ for $\alpha \geqslant \beta$. When $A_{1}=\Delta$ we obtain of course the standard Sobolev spaces which will be denoted as usual by $H_{\alpha}(\alpha \in \mathbb{R})$.

It will be essential in what follows to introduce the corresponding Besov spaces $\Lambda_{2, \infty}^{\alpha}(\alpha>0)\left(c f\right.$. [1]) which I shall denote for simplicity by $X_{\alpha}^{\infty}$. We have $f \in X_{\alpha}^{\infty}$ if

$$
\sup _{t} t^{-\alpha / 2}\left\|D_{\rho}(t) f\right\|_{X}<+\infty
$$

where we denote by $D_{\rho}(t)=t^{\rho} A^{\rho} \exp (-t A)$. The $\rho>0$ in the previous definition is immaterial as long as it is large enough (in fact $\rho>\alpha / 2$ ). I shall always assume that this is the case and drop the index $\rho$. Different choices of $\rho$ as above give equivalent norms ( $c f .[2],[3])$. The following two facts are vital for us

First we have $X_{\alpha} \subseteq X_{\alpha}^{\infty}(\alpha>0)$. Equivalently we have

$$
\|D(t) f\|_{X} \leqslant C t^{\alpha / 2}\|f\|_{\alpha}
$$

where here and in what follows $\|\cdot\|_{\alpha}$ denotes the norm in $X_{\alpha}$.

Second if we use the real interpolation method we obtain

$$
X_{\theta \alpha+(1-\theta) \beta}=\left[X_{\alpha}^{\infty}, X_{\beta}^{\infty}\right]_{\theta, 2}=\left[X_{\alpha}, X_{\beta}\right]_{\theta, 2}
$$

provided that $\alpha, \beta>0,0<\theta<1 . C f$. [1] [4] for the above facts. 
Let us now consider

$$
\Phi(t): C_{0}^{\infty}\left(\mathbb{R}^{n}\right) \rightarrow C^{\infty}\left(\mathbb{R}^{n}\right), \quad t>0,
$$

a family of mappings from $C_{0}^{\infty}$ to $C^{\infty}$ that depends on the positive parameter $(t>0)$. Notice that I have restricted myself, for simplicity, to our basic example $X=L^{2}\left(\mathbb{R}^{n}\right)$. The modifications in the abstract setting are obvious. Typical instances of $\Phi(t)$ in our applications will be

$$
\begin{aligned}
& \Phi(t)=T_{t}, \\
& \Phi(t)=T_{t}-I, \\
& \Phi(t)=T_{t} M-M T_{t}=\left[T_{t}, M\right], \\
& \Phi(t)=M_{1}\left[T_{t}, M\right]
\end{aligned}
$$

and also higher commutators. In the above examples $M, M_{1}: S \rightarrow \delta$ are continuous operators and $T_{t}=e^{-t B}$ is a continuous semigroup of operators that acts (i.e. is a continuous semigroup of bounded operators) on all (or the relevant) $X_{\alpha}$ spaces. We could for instance take $X_{\alpha}=H_{\alpha}$ the classical Sobolev spaces and take $T_{t}=e^{-t L}$ where $L$ is some pseudo-differential operator as above ( $c f$. [3]). In this context I shall adopt once and for all the following convention:

Semigroups $\left(T_{t}\right)_{t>0}$ as above will be substituted, when necessary, and without further notice, by the corresponding semigroups $\left(e^{-\lambda_{0} t} T_{t}\right)_{t>0}$ for appropriate $\lambda_{0}>0$ so that the behaviour at infinity will be controlled. This, equivalently, corresponds to replacing $L$ by $L+\lambda_{0}$ and, since all our considerations are local, it will have no effect in our theory.

I shall now consider the integral

$$
I_{\theta}=\int_{0}^{\infty} t^{\theta-1} \Phi(t) d t, \quad \theta \in \mathbb{C},
$$

(for the principal determination of $t^{\theta}$ with $\theta$ complex) and I shall immediately take advantage of my convention and assume that the above integral converges in norm at infinity. The issue will be the convergence of the above integral at 0 . The discussion that follows in the rest of this section will be devoted to this problem.

We shall assume that there exist two constants $A \in \mathbb{R}, B>0$ such that

$$
\|\Phi(t)\|_{\alpha_{0} \rightarrow \beta_{0}}=O\left(t^{A+B\left(\alpha_{0}-\beta_{0}\right)}\right), \quad t \rightarrow 0
$$

for some fixed $\alpha_{0}, \beta_{0} \in \mathbb{R}$. The notation $\|\bullet\|_{\alpha \rightarrow \beta}$ here and in what follows indicates the operator norm $X_{\alpha} \rightarrow X_{\beta}$. If we have $\operatorname{Re} \theta+A+B\left(\alpha_{0}-\beta_{0}\right)>0$ then the integral $I_{\theta}$ clearly converges at 0 . We shall consider therefore a $\theta_{0} \in \mathbb{C}$ for which

$$
\operatorname{Re} \theta_{0}+A+B\left(\alpha_{0}-\beta_{0}\right)=0 .
$$


We have quite generally for any $f \in C_{0}^{\infty}$ with $\|f\|_{\alpha}=1$

$$
\begin{aligned}
\left\|t^{-\beta / 2} D(t) I_{\theta} f\right\|_{X} \leqslant & t^{-\beta / 2} \int_{0}^{\infty} \xi^{\operatorname{Re} \theta-1}\|D(t) \Phi(\xi) f\|_{X} d \xi \\
= & t^{\left(\gamma_{1}-\beta\right) / 2} \int_{0}^{\lambda} \xi^{\operatorname{Re} \theta-1}\|\Phi(\xi) f\|_{\gamma_{1}} d \xi \\
& +t^{\left(\gamma_{2}-\beta\right) / 2} \int_{\lambda}^{\infty} \xi^{\operatorname{Re} \theta-1}\|\Phi(\xi) f\|_{\gamma_{2}} d \xi \\
= & I_{t}+I^{t},
\end{aligned}
$$

where we assume that $\beta, \gamma_{1}, \gamma_{2}>0$. I shall now make the stronger assumption that

$$
\|\Phi(t)\|_{\alpha \rightarrow \beta}=O\left(t^{A+B(\alpha-\beta)}\right), \quad t \rightarrow 0
$$

for $\alpha=\alpha_{0}$ and $\beta \in\left[\beta_{0}-\epsilon, \beta_{0}+\epsilon\right]$ for some positive $\epsilon>0$. With $\theta=\theta_{0}$, $\beta=\beta_{0}$ and $\gamma_{1}=\beta_{0}-\epsilon, \gamma_{2}=\beta_{0}+\epsilon$ in the above estimate we obtain

$$
\begin{array}{ll}
I_{t} \leqslant C t^{\left(\gamma_{1}-\beta_{0}\right) / 2} \lambda^{\operatorname{Re} \theta_{0}+A+B\left(\alpha_{0}-\gamma_{1}\right)} & \\
I^{t} \leqslant C t^{\left(\gamma_{2}+\beta_{0}\right) / 2}\left[\lambda^{\operatorname{Re} \theta_{0}+A+B\left(\alpha_{0}-\gamma_{2}\right)}+1\right], & \text { for } \lambda \leqslant 1, \\
I^{t} \leqslant C t^{\left(\gamma_{2}-\beta_{0}\right) / 2} \lambda^{-\epsilon_{0}}, & \text { for } \lambda>1 .
\end{array}
$$

Where we tacitly assume that

$$
\xi^{\operatorname{Re} \theta_{0}}\|\Phi(\xi)\|_{\alpha_{0} \rightarrow \gamma_{2}}=O\left(\xi^{-\epsilon_{0}}\right), \quad \xi \rightarrow \infty
$$

for some fixed $\epsilon_{0}>0$. (Our convention will take care of that in all our applications.) If we 'assume as we may that $\epsilon>0$ is small enough, and choose $\lambda=t^{1 / 2 B}$ we obtain from our hypotheses that $I_{t}+I^{t} \leqslant C$ for all $t>0$. This can be reinterpreted by saying that $I_{\theta_{0}}$ induces a bounded mapping. (I shall assume here that $\alpha_{0}, \beta_{0}>0$ )

$$
I_{\theta_{0}}=X_{\alpha_{0}} \rightarrow X_{\beta_{0}}^{\infty} .
$$

As a final step assume that (1.2) holds for $\alpha \in\left[\alpha_{0}-\epsilon, \alpha_{0}+\epsilon\right]$ and $\beta \in\left[\beta_{0}-\epsilon, \beta_{0}+\epsilon\right]$ for some $\epsilon>0$. The mapping $I_{\theta_{0}}$ is then bounded as in (1.3) for a whole range of $\alpha_{0}$ and $\beta_{0}$ around the central values. The interpolation theorem (1.1) implies therefore that

$$
I_{\theta_{0}}: X_{\alpha_{0}} \rightarrow X_{\beta_{0}}
$$

is a bounded mapping also.

The case $\alpha_{0}, \beta_{0}<0$ is handled by duality, since $X_{\alpha}^{*}=X_{-\alpha}$. We have to assume that the semigroup $T_{t}^{*}$ satisfies analogous conditions and we work with the analogous $\Phi^{*}(t)$. Once we have (1.4) with $\alpha_{0}, \beta_{0}>0$ and with $\alpha_{0}, \beta_{0}<0$ we interpolate and obtain (1.4) in general. 


\section{First Illustrations of the Real Interpolation Method}

Let $S(x, \xi) \in S_{1,0}^{2 m}(m>0)$ be real and let us assume that the operator $S(x, D)$ satisfies the sub-elliptic estimate

$$
\|f\|_{H_{m-\delta}}^{2}=\|f\|_{m-\delta}^{2} \leqslant C\left(\operatorname{Re}<S f, f>+\|f\|_{2}^{2}\right)
$$

for some $C>0$ and $0 \leqslant \delta \leqslant 1$. It is easy to see then that the semigroup $e^{-t S}=T_{t}$ acts continuously on all the classical Sobolev spaces $H_{\alpha}$. For $0 \leqslant \delta<1$ the proof is banal: To say that $e^{-t S}$ acts on $H_{\alpha}$ is equivalent to saying that $\exp \left(-t \Delta^{\alpha} S \Delta^{-\alpha}\right)$ acts on $L^{2}\left(\mathbb{R}^{n}\right)$ but the generator $\Delta^{\alpha} S \Delta^{-\alpha}$ is semibounded (i.e. $\operatorname{Re}(A f, f) \geqslant-C\|f\|_{2}^{2}$ ) by our sub-ellipticity condition and the standard commutation relation (cf. [5])

$$
\Delta^{\alpha} S \Delta^{-\alpha}-S \in O P S_{1,0}^{2 m-2} \bmod (\text { Skew-adjoined operators). }
$$

The above argument works even for $\delta=1$ provided that $m=1$. For more general $m>1$ we can still show that $S$ is semibounded on $H_{n}, n=1,2, \ldots$, provided that we give the following equivalent norm on $H_{n}$

$$
\|f\|_{H_{n}}^{*}=\left(\Delta^{n} f, f\right)^{1 / 2}+c_{1}\left(\Delta^{n-1} f, f\right)^{1 / 2}+\cdots+c_{p}\left(\Delta^{n-p} f, f\right)^{1 / 2}+\cdots
$$

for appropriate $c_{1}, c_{2}, \ldots, c_{n}>0$. At any rate this is not the issue here and let me simply assume that $e^{-t S}$ acts on all the $H_{\alpha}$ spaces $(\alpha \in \mathbb{R})$.

Because of the sub-ellipticity condition (2.1) we can identify $S$ with a closed Hilbert space operator and we can then define all the powers $S^{\sigma}(\sigma \in \mathbb{C})$ of $S$. The semigroup $e^{-t S}$ could for instance be used to define these powers (cf. [6]).

I shall illustrate here the results of Section 1 by showing that for every $\sigma \in \mathbb{C}$ with $\operatorname{Re} \sigma>0$ the operator $S^{\sigma}$ can be identified with a bounded operator

$$
\left(\lambda_{0}+S\right)^{\sigma}: H_{\sigma} \rightarrow H_{\alpha-2 m \operatorname{Re} \sigma}, \quad \alpha \in \mathbb{R},
$$

(for $\lambda_{0}>0$ large enough. Following my convention in Section 1, I shall drop the $\lambda_{0}$ from now on). It is clearly enough to prove this for $0<\operatorname{Re} \sigma<1$ where we have the formula ( $c f$. [6])

$$
S^{\sigma}=C_{\sigma} \int_{0}^{\infty} t^{-\sigma-1}\left(T_{t}-I\right) d t .
$$

Our theory in Section 1 with $X_{\alpha}=H_{\alpha}$ implies and the result (2.2) follows at once as soon as we show that $\Phi(t)=T_{t}-I$ satisfies

$$
\left\|T_{t}-I\right\|_{\alpha \rightarrow \alpha-2 m}=O(t), \quad t \rightarrow 0, \quad \alpha \in \mathbb{R},
$$

a fact that, by interpolation, once combined with the trivial bound

$$
\left\|T_{t}-I\right\|_{\alpha \rightarrow \alpha}=O(1)
$$


gives

$$
\left\|T_{t}-I\right\|_{\alpha \rightarrow \alpha-2 m \lambda}=O\left(t^{\lambda}\right), \quad t \rightarrow 0, \quad \alpha \in \mathbb{R}, \quad \lambda \in[0,1] .
$$

But (2.4) is of course evident. Indeed

$$
T_{t}-I=c \int_{0}^{t} S T_{\xi} d \xi
$$

and we only have to observe that $S \in O P S_{1,0}^{2 m}$ and that $T_{\xi}$ acts on the Sobolev spaces $H_{\alpha}$.

Negative powers can also be treated that way, in other words we can assume that $\operatorname{Re} \sigma<0$. Here we have to impose the condition that $0 \leqslant \delta<1$. For simplicity I shall also assume that $m \equiv 1$. (The results work in general but for our applications we shall only need the case $m=1$.) It was then shown in [3] that

$$
\left\|T_{t}\right\|_{\alpha \rightarrow \beta}=O\left(t^{(\alpha-\beta) / 2(1-\delta)}\right), \quad t \rightarrow 0,
$$

for all $\alpha \leqslant \beta$. The theory of Section 1 applies again (with $X_{\alpha}=H_{\alpha}$ ) and we deduce that

$$
S^{-\sigma}: H_{\alpha} \rightarrow H_{\alpha-2(1-\delta) \operatorname{Re} \sigma}, \quad \alpha \in \mathbb{R},
$$

for all $\sigma \in \mathbb{C}$ with $\operatorname{Re} \sigma>0$. This was proved in [3] with the same method.

The above methods give no information on the pure imaginary powers. In fact, we shall see in the next section that

$$
S^{i t}: H_{\alpha} \rightarrow H_{\alpha}, \quad \alpha, t \in \mathbb{R},
$$

is a bounded operator (since $S^{i t}$ is a group in $t$ the norm of that operator increases at most exponentially in $t \in \mathbb{R})$ as soon as the symbol $S(x, \xi) \geqslant 0$ is nonnegative. This fact together with complex interpolation theory (between «integer values» $\sigma=n+i t, n=0,1, \ldots)$ can be used to reprove (2.2).

\section{Duhamel's Formula, the Pure Imaginary Powers, and an Overview of the Ideas}

I shall introduce now some of the main ideas of this paper. For simplicity I shall first consider an operator that is self-adjoint and a sum of squares of fields

$$
L=\sum X_{j}^{*} X_{j}
$$

(a Hörmander operator) but on which I impose no sub-ellipticity conditions whatsoever (in other words I do not assume that the successive brackets of the fields $X_{j}$ span the tangent space). 
I shall consider the two semigroups

$$
T_{t}=e^{-t A}, \quad R_{t}=e^{-t B}, \quad A=(I+L), \quad B=\Lambda^{\alpha} A \Lambda^{-\alpha},
$$

where $\Lambda=(1+\Delta)^{1 / 2}$. «Duhamel's formula» then is the following observation

$$
\begin{aligned}
R_{t}-T_{t} & =\int_{0}^{t} \frac{d}{d s}\left(R_{s} T_{t-s}\right) d s \\
& =\int_{0}^{t} R_{s}(A-B) T_{t-s} d s .
\end{aligned}
$$

Using the standard commutation relations for pseudo-differentials we see that

$$
\begin{aligned}
A-B & =\sum_{j}\left(X_{j} E\right)+E \\
& =\sum_{j}\left(E X_{j}\right)+E
\end{aligned}
$$

where from here onwards, when confusion does not arise, I shall use the letter $E$ to indicate an arbitrary zero order in $O P S_{1,0}^{0}$ pseudo-differential (that can be different from place to place). We have therefore

$$
R_{t}-T_{t}=\sum_{j} \int_{0}^{t} R_{s} E X_{j} T_{t-s} d s+\int_{0}^{t} R_{s} E T_{t-s} d s .
$$

In the above decomposition the last term is harmless. It has an $L^{2} \rightarrow L^{2}$ norm (in fact an $L^{p} \rightarrow L^{p}$ norm $c f$. [3] $1<p<+\infty$ ) that is $O(t)$. This is simply because both $T_{t}$ and $R_{t-s}$ (by Section 2) are $L^{2}$ (in fact $L^{p}$ ) bounded uniformly for $0<t<1$ and so is $E \in S_{1,0}^{0}$.

The terms

$$
\int_{0}^{t} R_{s} E X_{j} T_{t-s} d s=\int_{0}^{t} R_{s} E X_{j} A^{-1 / 2} A^{1 / 2} T_{t-s} d s
$$

are more subtle. Indeed we have:

$$
\left\|A^{1 / 2} e^{-t A}\right\|_{L^{2} \rightarrow L^{2}}=O\left(t^{-1 / 2}\right) \quad t \rightarrow 0,
$$

and clearly

$$
\left\|X_{j} f\right\|_{L^{2}} \leqslant\left\|A^{1 / 2} f\right\|_{L^{2}} \quad j=1,2, \ldots
$$

This means that $\left\|X_{j} A^{-1 / 2}\right\|_{L^{2} \rightarrow L^{2}} \leqslant C$. Putting all these remarks together we see that we can estimate the $\|\cdot\|_{L^{2} \rightarrow L^{2}}$ norm of the terms in (3.1) by

$$
c \int_{0}^{t} \frac{d s}{(t-s)^{1 / 2}}=O\left(t^{1 / 2}\right) \text {. }
$$


In fact the $\|\bullet\|_{L^{p} \rightarrow L^{p}}(1<p<+\infty)$ of these terms can also be estimated by $O\left(t^{1 / 2}\right)$ by the same method. This however is more delicate since it depends on the $L^{p} \rightarrow L^{p}$ estimate of the singular integral transform: $X_{j} L^{-1 / 2}$ (cf. [7], [8], [9]). This is only known to hold for a sub-elliptic operator $L$. I shall not press the matter further at this stage.

Another incidental fact that we notice at this stage is that the above estimate automatically implies that

$$
\left\|T_{t}-R_{t}\right\|_{\alpha \rightarrow \alpha}=O\left(t^{1 / 2}\right)
$$

(just take differences!) where $\|\cdot\|_{\alpha \rightarrow \beta}$ are operator norms between the classical Sobolev spaces $H_{\alpha}$. Furthermore, writing

$$
T_{2 t}-R_{2 t}=T_{t}\left(T_{t}-R_{t}\right)+\left(T_{t}-R_{t}\right) R_{t}
$$

allows us to conclude that for a $\delta$-sub-elliptic operator with $\delta<1$ (i.e. (2.1) is verified for $m=1$ and that $\delta$ ) we have: (we use (2.5) which is then automatically also valued for $R_{t}$ )

$$
\left\|T_{t}-R_{t}\right\|_{\alpha \rightarrow \beta}=O\left(t^{1 / 2+(\alpha-\beta) / 2(1-\delta)}\right), \quad t \rightarrow 0,
$$

for every $\beta \geqslant \alpha$. One can also show by an easy modification of the above argument, that for $\beta \in[\alpha-1, \alpha]$ we have in (3.3) the bound $O\left(t^{1 / 2+(\alpha-\beta) / 2}\right)$. These remarks are not essential however here, they are merely meant to illustrate the ideas involved.

Let us now recall the standard formula valid for any semigroup generator $D$ and $\epsilon>0, s \in \mathbb{R}$

$$
D^{-\epsilon+i s}=C(\epsilon, s) \int_{0}^{\infty} t^{\epsilon-1+i s} e^{-t D} d t .
$$

Let us fix $s \neq 0$ and decompose

$$
\int_{0}^{\infty} t^{\epsilon-1+i s} R_{t} d t=\int_{0}^{\infty} t^{\epsilon-1+i s} T_{t} d t+\int_{0}^{\infty} t^{\epsilon-1+i s}\left(R_{t}-T_{t}\right) d t .
$$

The second integral can be made to converge absolutely and uniformly as $\epsilon \rightarrow 0$ in the norm $\|\cdot\|_{L^{2} \rightarrow L^{2}}$. This is because of (3.3) and our standing convention of Section 1 about $t \rightarrow \infty$. The first integral expresses $A^{-\epsilon+i s}$ and by the spectral theorem on the spectral decomposition of $L$ it converges strongly to the unitary operator $A^{i s}$. Our conclusion is that $\Lambda^{\alpha} A^{i s} \Lambda^{-\alpha}$ is an $L^{2} \rightarrow L^{2}$ bounded operator which is our required result. In fact, since the convention of Section 1 was used to control the infinity of the above integrals, the way we have written (2.7) is not correct. What is correct is

$$
\left(\lambda_{0}+L\right)^{i s}: H_{\alpha} \rightarrow H_{\alpha}
$$


is bounded for $\alpha, s \in \mathbb{R}$ for some (in fact, all) $\lambda_{0}>0$. I will however systematically ignore the addition of positive constants and continue to write a formula like (3.5) in the slightly incorrect but more convenient form (2.7). Observe finally that $e^{-t A}$ acts holomorphically on each $H_{\alpha}$ space. Indeed, consider for some appropriate $\lambda_{0}>0, \exp \left(-\lambda_{0} z-z A\right)=U(z)(\operatorname{Re} z>0)$ so that $\|U(z)\|_{L^{2} \rightarrow L^{2}} \leqslant C$ for $\operatorname{Re} z>0$ and $\|U(z)\|_{\alpha \rightarrow \alpha} \leqslant C$ when $z$ lies on the real axis. By an appropriate conformal mapping we can re-express this in the form

$$
\left\|\Lambda^{\alpha \omega / 2} U(w) \Lambda^{-(\alpha \omega / 2)}\right\|_{L^{2} \rightarrow L^{2}} \leqslant C
$$

when $\operatorname{Re} w=0$ and $\operatorname{Re} w=1$ (here $0 \leqslant \operatorname{Re} w \leqslant 1$ ) and Phragmèn-Lindelöf does the rest ( $c f .[20])$.

Alternatively, we can use the action of $A^{i s}$ on $H_{\alpha}$ to interpolate the information on $\left\|(t A)^{z} e^{-t A}\right\|_{\alpha \rightarrow \alpha}$ for $\alpha=r \in \mathbb{R}, \operatorname{Re} z=0$ and $\alpha=0, \operatorname{Re} z=1,2, \ldots$.

In the same spirit if we use complex interpolations between the $H_{\alpha}^{p}$ spaces $(1 \leqslant p \leqslant+\infty)$

$$
H_{\alpha}^{p}=\left\{f: \Lambda^{\alpha / 2} f \in L^{p}\right\}
$$

we see that

$$
L^{i s}: H_{\alpha}^{p} \rightarrow H_{\alpha}^{p}
$$

for $1<p<+\infty, \alpha \in \mathbb{R}$. The other non trivial information used here is the fact that

$$
(I+L)^{i s}: L^{p} \rightarrow L^{p}
$$

is bounded for $1<p<+\infty$ (cf. [20]).

Before we move on, we shall address ourselves the question whether the above considerations generalize to more general self-adjoint differential operators

$$
L=h^{-1} \sum_{i, j}\left(\partial_{i} a_{i j} h \partial_{j}\right)
$$

with positive characteristic $\left(a_{i j}\right) \geqslant 0, h, h^{-1} \in C^{\infty}$ or better still for pseudodifferentials with positive symbol $\rho(x, \xi) \geqslant 0$

$$
L=\rho(x, D)+\rho^{*}(x, D)+C
$$

for appropriate positive $C>0$.

The answer is yes in the most general case (3.7) for $L^{2}$ norms. Everything we said about $L^{p}$ norms for $(p \neq 2)$ also holds in the case (3.6). But to see all this it is difficult and totally alien with the methods of this paper. It will be presented in a second instalment of this work. 


\section{Commutators with $E \in S_{1,0}^{0}$}

Let us get back now to the main theme of this paper. I shall fix from here onwards the following notation

$$
A=I+L=I+\sum X_{j}^{*} X_{j}
$$

for $C^{\infty}$ fields $X_{j}$ and I shall also denote as in Section 1 by

$$
X_{\alpha}=\left\{f: A^{\alpha / 2} f\right\}, \quad \alpha \in \mathbb{R}
$$

the corresponding Sobolev spaces. (No confusion will arise, I am sure, between the fields $X_{j}$ and the spaces $X_{\alpha}$ !)

Let $E \in O P S_{1,0}^{0}$ be an arbitrary zero order pseudo-differential. By standard commutation we have as before

$$
[A, E]=A E-E A=\sum_{j}\left(E X_{j}\right)+E
$$

(for different $E$ 's in $O P S_{1,0}^{0}$ ) this means that

$$
\begin{aligned}
\|[A, E] f\|_{L^{2}} & \leqslant \sum_{j}\left\|E X_{j} f\right\|_{L^{2}}+\|E f\|_{L^{2}} \\
& \leqslant C \sum\left\|X_{j} f\right\|_{L^{2}}+\|f\|_{L^{2}} \\
& \leqslant C\left\|A^{1 / 2} f\right\|_{L^{2}}
\end{aligned}
$$

(observe, and, this will be a recurrent point in what follows, that $\left\|A^{1 / 2} f\right\|_{L^{2}}$ $\geqslant C\|f\|_{L^{2}}$ ). Considerably more is in fact true.

We shall assume that $L$ is sub-elliptic i.e. that the sucessive brackets of the fields $X_{j}$ span at every point the tangent space. The use of sub-ellipticity that we shall make at this stage is that it implies the estimate

$$
\left\|X_{i_{1}} X_{i_{2}} \cdots X_{i_{k}} f\right\|_{L^{2}} \leqslant C\left\|A^{k / 2} f\right\|_{L^{2}}, \quad f \in C_{0}^{\infty}
$$

for $k=1,2, \ldots$. This is a deep result and unlike (3.2) (for $k=1$ ) it is not just a consequence of a formal identity. There are many ways of seeing this and they all depend on singular integral theory ( $c f$. [9], p. 96). My favourite way of seeing it runs like this: First prove (4.2) for a nilpotent Lie group and «constant» fields (i.e. elements of the Lie algebra, $c f$. [21]). Then use the parametrix and the lifting theorem of [22]. Be it as it may, we are going to take (4.2) for granted here.

Using successive commutations again and developing $A^{k}(k=1,2, \ldots)$ as sums of products of fields we see that (4.2) implies (observe that $\left\|A^{j} f\right\|$ is an increasing function of $j \in \mathbb{R}$ ) that 


$$
\begin{aligned}
\left\|\left[A^{k}, E\right] f\right\|_{L^{2}} & \leqslant C\left\|A^{k-1 / 2} f\right\|_{L^{2}} \\
\left\|A^{n}\left[A^{k}, E\right] f\right\|_{L^{2}} & \leqslant C\left\|A^{n+k-1 / 2} f\right\|_{L^{2}}
\end{aligned}
$$

for $k, n=1,2, \ldots$ But, denoting from here onwards by $\|\cdot\|_{\alpha}=\|\cdot\|_{X_{\alpha}}$, this simply says that

$$
\left\|\left[A^{k}, E\right]\right\|_{2 n+(2 k-1) \rightarrow 2 n} \leqslant C
$$

which by interpolation implies that

$$
\left\|\left[A^{k}, E\right]\right\|_{\alpha \rightarrow \alpha-2 k+1} \leqslant C
$$

for all $\alpha \in \mathbb{R}$, and $\alpha$ large enough.

If we dualize the above statement (observe that $X_{\alpha}^{*}=X_{-\alpha}$ ) and then interpolate again we finally obtain that (4.3) holds for all $\alpha \in \mathbb{R}, k=0,1,2, \ldots$. In particular we have

$$
\|[A, E]\|_{\alpha+1 \rightarrow \alpha} \leqslant C
$$

for all $\alpha \in \mathbb{R}$. Our next task will be to prove the following more general and basic fact

$$
\left\|\left[A^{\sigma}, E\right]\right\|_{\alpha \rightarrow \alpha-2 \operatorname{Re} \sigma+1} \leqslant C
$$

for any $\sigma \in \mathbb{C}$. (4.5) follows automatically for $\operatorname{Re} \sigma<1$ from the theory of Section 1 as soon as we have shown the estimate

$$
\left\|\left[e^{-t A}, E\right]\right\|_{\alpha \rightarrow \beta}=O\left(t^{1 / 2+(\alpha-\beta) / 2}\right), \quad t \rightarrow 0,
$$

for $\alpha, \beta \in \mathbb{R}$ with $\beta \geqslant \alpha-1$. Indeed it suffices to insert in the $I_{\theta}$ of Section 1 the two formulas (2.3), (3.4) and to observe that $\left[e^{-t A}-I, E\right]=\left[e^{-t A}, E\right]$. To prove (4.6) we first observe that

$$
\begin{aligned}
{\left[E, e^{-2 t A}\right] } & =e^{-t A}\left[E, e^{-t A}\right]+\left[E, e^{-t A}\right] e^{-t A} \\
{\left[E, e^{-t A}\right] } & =\int_{0}^{t} \frac{d}{d s}\left(e^{-(t-s) A} E e^{-s A}\right) d s \\
& =\int_{0}^{t} e^{-(t-s) A}[E, A] e^{-s A} d s
\end{aligned}
$$

which together give

(4.7) $\left[E, e^{-2 t A}\right]=\int_{0}^{t} e^{-(2 t-s) A}[E, A] e^{-s A} d s+\int_{0}^{t} e^{-(t-s) A}[E, A] e^{-(s+t) A} d s$. 
(In all these formulas, and they will be many, I tend to ignore irrelevant \pm signs.) We shall now «factorize» the $\left\|\left[E, e^{-2 t A}\right]\right\|_{\alpha \rightarrow \beta}$ inside the integrals and estimate it by

$$
\begin{aligned}
\int_{0}^{t}\left\|e^{-(2 t-s) A}\right\|_{\alpha-1 \rightarrow \beta}\|[E, A]\|_{\alpha \rightarrow \alpha-1}\left\|e^{-s A}\right\|_{\alpha \rightarrow \alpha} d s & \\
& +\int_{0}^{t}\left\|e^{-(t-s) A}\right\|_{\beta \rightarrow \beta}\|[E, A]\|_{\beta+1 \rightarrow \beta}\left\|e^{-(s+t) A}\right\|_{\alpha \rightarrow \beta+1} d s .
\end{aligned}
$$

$\|[E, A]\|_{\alpha \rightarrow \alpha-1}$ is of course bounded by (4.4) and we also have $\left\|e^{-s A}\right\|_{\alpha \rightarrow \beta}=$ $O\left(s^{(\alpha-\beta) / 2}\right)$ for $\beta \geqslant \alpha$, because of the holomorphic action of $e^{-t A}$ on $L^{2}$. We see therefore that the two integrals can be estimated respectively by

$$
\begin{aligned}
\int_{0}^{t}(2 t-s)^{(\alpha-1-\beta) / 2} d s=O\left(t^{1 / 2+(\alpha-\beta) / 2}\right), & \beta \geqslant \alpha-1 \\
\int_{0}^{t}(t+s)^{(\alpha-1-\beta) / 2} d s=O\left(t^{1 / 2+(\alpha-\beta) / 2}\right), & \beta \geqslant \alpha-1 .
\end{aligned}
$$

The estimate (4.6) and the estimate (4.5) for $\operatorname{Re} \sigma<1$ follow. To obtain (4.5) for arbitrary $\sigma \in \mathbb{C}$ we just use (4.3) and formula

$$
\left[A^{k+\sigma}, E\right]=A^{k}\left[A^{\sigma}, E\right]+\left[A^{k}, E\right] A^{\sigma}
$$

with $k=1,2, \ldots \operatorname{Re} \sigma<0$ and observe that for arbitrary $\sigma \in \mathbb{C}, A^{\sigma}: X_{\alpha} \rightarrow$ $X_{\alpha-2 \operatorname{Re} \sigma}$ is an isomorphism.

The estimate (4.5) is a special case of the following more general result

$$
\left\|\left[\cdots\left[\left[A^{\sigma}, E_{1}\right], E_{2}\right] \cdots, E_{k}\right]\right\|_{\alpha \rightarrow \alpha-2 \operatorname{Re} \sigma+k} \leqslant C
$$

for arbitrary $\alpha \in \mathbb{R}, \sigma \in \mathbb{C}, k=1,2, \ldots$ where $E_{1}, \ldots \in O P S_{1,0}^{0}$ are zero order pseudo-differentials. I shall give below the proof for $k=2$. (The general case is similar and we shall have no immediate use of anyway.)

We start with the formula (4.7) where $E=E_{1}$ and take the commutator with $E_{2}$ so as to obtain [[ $\left.\left.e^{-2 t A}, E_{1}\right], E_{2}\right]$. We insert the commutator with $E_{2}$ inside each integral so as to obtain six different terms. The first three are

$$
\begin{aligned}
& I_{1}=\int_{0}^{t}\left[e^{-(2 t-s) A}, E_{2}\right]\left[A, E_{1}\right] e^{-s A} d s, \\
& I_{2}=\int_{0}^{t} e^{-(2 t-s) A}\left[\left[A, E_{1}\right], E_{2}\right] e^{-s A} d s, \\
& I_{3}=\int_{0}^{t} e^{-(2 t-s) A}\left[A, E_{1}\right]\left[e^{-s A}, E_{2}\right] d s .
\end{aligned}
$$

The other three terms $I_{1}^{\prime}, I_{2}^{\prime}, I_{3}^{\prime}$ are identical except that $2 t-s$ on the first exponential is replaced by $t-s$ and the $s$ in the second exponential is replaced by $s+t$. 
We shall first analyse the terms $I_{1}$ and $I_{3}$, we have

$$
\left\|I_{1}\right\|_{\alpha \rightarrow \beta} \leqslant \int_{0}^{t}\|\cdot\|_{\alpha-1 \rightarrow \beta}\|\cdot\|_{\alpha \rightarrow \alpha-1}\|\cdot\|_{\alpha \rightarrow \alpha} d s .
$$

The factors are written of course in the same order and

$$
\|\cdot\|_{\alpha-1 \rightarrow \beta}=O\left((2 t-s)^{1 / 2+(\alpha-1-\beta) / 2}\right)
$$

by our estimate (4.6) for $\beta \geqslant \alpha-2$. For $\beta \geqslant \alpha-2$ we have thus $\left\|I_{1}\right\|_{\alpha \rightarrow \beta}=$ $O\left(t^{1+(\alpha-\beta) / 2}\right)$. Similarly we factorize

$$
\left\|I_{3}\right\|_{\alpha \rightarrow \beta} \leqslant \int_{0}^{t}\|\cdot\|_{\alpha-2 \rightarrow \beta}\|\cdot\|_{\alpha-1 \rightarrow \alpha-2}\|\cdot\|_{\alpha \rightarrow \alpha-1} d s .
$$

The term $\|\cdot\|_{\alpha \rightarrow \alpha-1}=O(s)$ because of (4.6), $\|\cdot\|_{\alpha-1 \rightarrow \alpha-2} \leqslant C$ and $\|\cdot\|_{\alpha-2 \rightarrow \beta}$ $=O\left((2 t-s)^{(\alpha-\beta) / 2-1}\right)$ for $\beta \geqslant \alpha-2$. We obtain thus $\left\|I_{3}\right\|_{\alpha \rightarrow \beta}=O\left(t^{1+(\alpha-\beta) / 2}\right)$ for $\beta \geqslant \alpha-2$.

To estimate the middle term we have to phase some new phenomena that give us a glimpse of what happens in (4.8) when $k \geqslant 2$ is arbitrary. We factor again

$$
\left\|I_{2}\right\|_{\alpha \rightarrow \beta}=\int_{0}^{t}\|\cdot\|_{\gamma \rightarrow \beta}\|\cdot\|_{\alpha \rightarrow \gamma}\|\cdot\|_{\alpha \rightarrow \alpha} d s
$$

and we may as well assume that $\beta \geqslant \alpha-2$ since this condition is imposed on us by the other two terms. We distinguish two cases:

Case (i): $\beta \geqslant \alpha$. We then set $\gamma=\alpha$ and we shall show that the middle term satisfies

$$
\left\|\left[\left[A, E_{1}\right], E_{2}\right]\right\|_{\alpha \rightarrow \alpha} \leqslant C .
$$

Assume this for the moment. Then the first term gives $\|\cdot\|_{\gamma \rightarrow \beta}=(2 t-s)^{(\alpha-\beta) / 2}$ and we obtain $\left\|I_{2}\right\|_{\alpha \rightarrow \beta}=O\left(t^{1+(\alpha-\beta) / 2}\right)$ as before.

Case (ii): $\beta \in[\alpha-2, \alpha]$. We then set $\gamma=\alpha-2$ and from (4.10) it follows a fortiori (since $X_{\alpha} \subset X_{\gamma}$ ) that the middle term in (4.9) satisfies $\|\bullet\|_{\alpha \rightarrow \gamma} \leqslant C$. The first term satisfies then \|\|$_{\gamma \rightarrow \beta}=O(2 t-s)^{(\alpha-\beta) / 2-1}$ so that we obtain then $\left\|I_{2}\right\|_{\alpha \rightarrow \beta}=O\left(t^{(\alpha-\beta) / 2}\right.$ ) which for $\beta=\alpha-2$ gives $\left\|I_{2}\right\|_{\alpha \rightarrow \alpha-2}=O(t)$. But since by our previous case we have $\left\|I_{2}\right\|_{\alpha \rightarrow \alpha}=O(t)$ it follows that $\left\|I_{2}\right\|_{\alpha \rightarrow \beta}=O(t)$ for all $\beta \in[\alpha-2, \alpha]$. We can unify all the above and simply say that

$$
\left\|I_{1}+I_{2}+I_{3}\right\|_{\alpha \rightarrow \beta}=O\left(\max \left\{t, t^{1+(\alpha-\beta) / 2}\right\}\right)
$$

for $\beta \geqslant \alpha-2$, (which in $O(t)$ if $\beta \leqslant \alpha$ ).

It remains to prove (4.10) which is a special case of the following more general

$$
\left\|\left[\left[A^{k}, E_{1}\right], E_{2}\right]\right\|_{\alpha \rightarrow \alpha-2 k+2} \leqslant C
$$

valid for $k=1,2, \ldots$. 
The estimate (4.11) is proved exactly as in (4.3) provided that $\alpha=2 n$ is an even integer. Indeed (4.11) simply says then that

$$
\left\|A^{n}\left[\left[A^{k}, E_{1}\right], E_{2}\right]\right\|_{L^{2}} \leqslant C\left\|A^{n+k-1} f\right\|_{L^{2}}
$$

and we use pseudo-differential calculus and the basic singular integral estimate (4.2). (In the development of the commutators $\left[\left[A^{k}, E_{1}\right] E_{2}\right] t w o$ fields $X_{i_{1}}$ and $X_{i_{2}}$ are knocked out. This is done because either two commutators of the form $\left[X_{i_{1}}, E_{1}\right]$ and $\left[X_{i_{2}}, E_{1}\right]$ appear. These commutators then convert these fields into $S_{1,0}^{0}$ operators. Or only one such commutator appears but then we must have a term of the form $\left[E_{1}, E_{2}\right] \in S_{1,0}^{-1}$ that combines with some $X_{i_{\alpha}} \in S_{1,0}^{1}$ to give an element in $S_{1,0}^{0}$.) To finish the proof of (4.12) for arbitrary $\alpha \in \mathbb{R}$, i.e. we dualize and interpolate.

We are left with the other three terms $I_{1}^{\prime}, I_{2}^{\prime}, I_{3}^{\prime}$. The discussion is entirely analogous and will be left for the reader. The important point is that we now have to perform the «jump» from $\alpha$ to $\beta$ at the term $e^{-(t+s) A}$. Before we made that jump at the term $e^{-(2 t-s) A}$. This is to make sure that the integrals converge at the two ends.

Putting everything together we finally obtain

$$
\left\|\left[\left[e^{-t A}, E_{1}\right], E_{2}\right]\right\|_{\alpha \rightarrow \beta}=O\left(t^{1+(\alpha-\beta) / 2}\right)
$$

for all $\beta \geqslant \alpha$.

We are finally in a position to give the proof of (4.8) for $k=2$. Indeed the theory of Section 1 together with (4.13) and formula (3.4) allows us to conclude that

$$
\left\|\left[\left[A^{\sigma}, E_{1}\right], E_{2}\right]\right\|_{\alpha \rightarrow \alpha-2 \operatorname{Re} \sigma+2} \leqslant C
$$

for all $\alpha \in \mathbb{R}, \sigma \in \mathbb{C}, \operatorname{Re} \sigma<1$. To obtain the result for arbitrary $\sigma \in \mathbb{C}$ we develop

$$
\begin{aligned}
{\left[\left[A^{\sigma+k}, E_{1}\right], E_{2}\right]=} & {\left[\left[A^{\sigma}, E_{1}\right], E_{2}\right] A^{k}+\left[A^{\sigma}, E_{1}\right]\left[A^{k}, E_{2}\right] } \\
& +\left[A^{\sigma}, E_{2}\right]\left[A^{k}, E_{1}\right]+A^{\sigma}\left[\left[A^{k}, E_{1}\right], E_{2}\right]
\end{aligned}
$$

with $k=1,2, \ldots ; \operatorname{Re} \sigma<1$. It is then only a matter of combining (4.11), (4.5) and our previous result (4.14) and we are done.

\section{The Conjugation Operation $c_{\lambda}$}

This section runs parallel to the previous one. Here we shall consider expressions of the form

$$
A-\Lambda^{s} A \Lambda^{-s}=\Lambda^{s}\left[A, \Lambda^{-s}\right]=\left[\Lambda^{s}, A\right] \Lambda^{-s}, \quad s \in \mathbb{R},
$$


with $\Lambda=(1+\Delta)^{1 / 2}$ and the standard Laplacian $\Delta$. I shall also denote $c_{s}(A)=\Lambda^{s} A \Lambda^{-s}$ so that we have

$$
\Lambda^{s_{1}}\left[A, \Lambda^{-s}\right] \Lambda^{s_{2}}=c_{\lambda}(A)-c_{\mu}(A) .
$$

For $s_{1}+s_{2}=s$ and appropriate $\lambda, \mu \in \mathbb{R}$.

The operator $A$, the spaces $X_{\alpha}(\alpha \in \mathbb{R})$ and the norms $\|\cdot\|_{\alpha}=\|\cdot\|_{X_{\alpha}}$ have the same meaning as before. It is also clear that with $s_{1}+s_{2}=s$ we have

$$
\left\|\Lambda^{s_{1}}\left[A, \Lambda^{-s}\right] \Lambda^{s_{2}} f\right\|_{L^{2}} \leqslant C\left\|A^{1 / 2} f\right\|_{L^{2}} .
$$

Similarly if we impose sub-ellipticity on $A$ we obtain by the singular integral theorem and commutations of pseudo-differentials that

$$
\left\|c_{\lambda}\left(A^{k}\right)-c_{\mu}\left(A^{k}\right)\right\|_{\alpha \rightarrow \alpha-2 k+1} \leqslant C
$$

for arbitrary $\alpha \in \mathbb{R}$, and $k=1,2, \ldots$.

Our first aim is to prove the analogue of (4.5)

$$
\left\|\Lambda^{s_{1}}\left[A^{\sigma}, \Lambda^{-s}\right] \Lambda^{s_{2}}\right\|_{\alpha \rightarrow \beta}=\left\|c_{\lambda}\left(A^{\sigma}\right)-c_{\mu}\left(A^{\sigma}\right)\right\|_{\alpha \rightarrow \beta} \leqslant C
$$

for $\beta=\alpha-2 \operatorname{Re} \sigma+1, \alpha \in \mathbb{R}, \sigma \in \mathbb{C}$ (and $s_{1}+s_{2}=s$ of course). It is clearly enough (take differences) to assume $\mu=0$ and consider $\left(I-c_{\lambda}\right)\left(A^{\sigma}\right)=A^{\sigma}$ - $B A^{\sigma} B^{-1}$ with $B$ some real power of $\Lambda$.

Just as in Section 4 to show (5.2) it is enough to prove the following estimate

$$
\left\|\left(I-c_{\lambda}\right)\left(e^{-t A}\right)\right\|_{\alpha \rightarrow \beta}=O\left(t^{1 / 2+(\alpha-\beta) / 2}\right), \quad t \rightarrow 0,
$$

for $\beta \geqslant \alpha-1$. This implies in particular that

$$
\left\|c_{\lambda}\left(e^{-t A}\right)\right\|_{\alpha \rightarrow \beta}=O\left(t^{(\alpha-\beta) / 2}\right), \quad t \rightarrow 0,
$$

for $\beta \geqslant \alpha$. Indeed (5.4) is a consequence of the holomorphicity of $e^{-t A}$ on $X$ for $\lambda=0$ and it follows for general $\lambda$ by this special case and (5.3). The estimate (5.3) by the same method implies the result (5.2) for $\operatorname{Re} \sigma<1$.

But then if we combine this special case $\operatorname{Re} \sigma<1$ together with the special case $\sigma=k=1,2, \ldots$ of $(5.1)$ and the identity

$$
\left(1-c_{\lambda}\right)\left(A^{k+\sigma}\right)=A^{\sigma}\left(1-c_{\lambda}\right)\left(A^{k}\right)+\left[\left(1-c_{\lambda}\right)\left(A^{\sigma}\right)\right] c_{\lambda}\left(A^{k}\right)
$$

we obtain the estimate (5.2) in general. Observe here that

(5.5) $\left\|C_{\lambda}\left(A^{\rho}\right)\right\|_{\alpha \rightarrow \alpha-2 \operatorname{Re} \rho} \leqslant\left\|A^{\rho}\right\|_{\alpha \rightarrow \alpha-2 \operatorname{Re} \rho}+\left\|\left(1-c_{\lambda}\right) A^{\rho}\right\|_{\alpha \rightarrow \alpha-2 \operatorname{Re} \rho} \leqslant C$.

(Alternative pseudo-differential calculus and the sub-elliptic estimate (4.2) can be used directly to control $c_{\lambda}\left(A^{k}\right)$ for $k=1,2, \ldots$.) 
The proof of (5.3) is done in two steps. Let $c_{\lambda}\left(e^{-t A}\right)=B e^{-t A} B^{-1}=R_{t}$. The first step consists in showing that (5.4) holds for $\beta \in[\alpha, \alpha+1[, \alpha \in \mathbb{R}$. Towards that let

$$
\begin{aligned}
\phi(t) & =e^{-t A}-R_{t} \\
& =\int_{0}^{t} e^{-(t-s) A} B\left[A, B^{-1}\right] R_{s} d s \\
& =\int_{0}^{t} e^{-(t-s) A} B\left[A, B^{-1}\right] \phi(s)+I(t), \\
I(t) & =\int_{0}^{t} e^{-(t-s) A} B\left[A, B^{-1}\right] e^{-s A} d s .
\end{aligned}
$$

We shall first estimate $I(t)$,

$$
\|I(t)\|_{\alpha \rightarrow \beta} \leqslant \int_{0}^{t}\left\|e^{-(t-s) A}\right\|_{\gamma-1 \rightarrow \beta}\left\|B\left[A, B^{-1}\right]\right\|_{\gamma \rightarrow \gamma-1}\left\|e^{-s A}\right\|_{\alpha \rightarrow \gamma} d s
$$

we shall choose $\gamma \geqslant \alpha, \beta \geqslant \gamma-1$ so that the above integral can be estimated by

$$
\int_{0}^{t}(t-s)^{(\gamma-1-\beta) / 2} s^{(\alpha-\gamma) / 2} d s=O\left(t^{1 / 2+(\alpha-\beta) / 2}\right)
$$

as long as $\gamma-1-\beta>-2, \alpha-\gamma>-2$. All in all, for the above to work we need $\gamma \in[\alpha, \alpha+2[\cap] \beta-1, \beta+1]=a$, and $a \neq \varnothing$ if and only if $\beta \in[\alpha-1, \alpha+3[$. In the range $\beta \in\left[\alpha-1, \alpha+3\right.$ [ we have therefore the estimate $\|I(t)\|_{\alpha \rightarrow \beta}=$ $O\left(t^{1 / 2+(\alpha-\beta) / 2}\right)$.

If we inject that information in the formula (5.6) and set $\psi(t)=\|\phi(t) f\|_{\beta}$ for some $f \in C_{0}^{\infty}$ in the unit ball of $X_{\alpha}$, we see that

$$
\psi(t) \leqslant C \int_{0}^{t}\left\|e^{-(t-s) A} B\left[A, B^{-1}\right]\right\|_{\beta \rightarrow \beta} \psi(s) d s+O\left(t^{1 / 2+(\alpha-\beta) / 2}\right) .
$$

Taking the adjoint of the operator inside \|\|$_{\beta \rightarrow \beta}$ and using (5.1) we see that it satisfies $\|\cdot\|_{\beta \rightarrow \beta}=O\left((t-s)^{-1 / 2}\right)$. We end up thus with the following integral inequality

$$
\psi(t) \leqslant C t^{1 / 2+(\alpha-\beta) / 2}+C \int_{0}^{t} \psi(t-s) s^{-1 / 2} d s .
$$

Now if $f \in C_{0}^{\infty} \psi(t)$ is (semi-)continuous and $\psi(0)=0$, so if $t_{0}$ is the first value of $t$ for which $\psi(t) \geqslant 1$ we have $1 \leqslant c t_{0}^{1 / 2+(\alpha-\beta) / 2}+c t_{0}^{1 / 2}$ and therefore $t_{0} \geqslant c>0$ if $\beta \in[\alpha-1, \alpha+1[$.

This simply says that $\psi(t)=O(1)$ uniformly in $f$. Injecting this information in (5.8) gives

$$
\psi(t)=O\left(t^{1 / 2+(\alpha-\beta) / 2}+t^{1 / 2}\right), \quad \beta \in[\alpha-1, \alpha+1[.
$$


That information could be reinjected in (5.8) to obtain a more precise result on $\psi(t)$. This however is unnecessary at this stage. All we need is to combine (5.9) with the estimate $\left\|e^{-t A}\right\|_{\alpha \rightarrow \beta}=O\left(t^{(\alpha-\beta) / 2}\right)(\beta \geqslant \alpha)$ and we deduce (5.4) in the range $\beta \in[\alpha, \alpha+1[$.

At this point we start from scratch. We shall assume (by induction) that (5.4) holds in the range $\beta \in[\alpha, \alpha+k$ [ for some integer $k=1,2, \ldots$ and we shall prove the inductive step that then (5.4) holds in fact in the range $\beta \in[\alpha, \alpha+k+1[$. (This easily implies that (5.3) holds in the range $[\alpha-1, \alpha+k+1$ [.) Once this is done the proof of both (5.3) and (5.4) is completed for their full range. Towards the proof of this inductive step we proceed as follows

We have

$$
e^{-2 t A}-R_{2 t}=\int_{0}^{t} e^{-(2 t-s) A} B\left[A, B^{-1}\right] R_{s} d s+\int_{0}^{t} e^{-(t-s) A} B\left[A, B^{-1}\right] R_{s+t} d s .
$$

It follows that we can estimate the norm $\left\|e^{-t A}-R_{t}\right\|_{\alpha \rightarrow \beta}$ by the sum of the following two integrals,

$$
\begin{aligned}
& \int_{0}^{t}\left\|e^{-(2 t-s) A}\right\|_{\alpha-1 \rightarrow \beta}\left\|B\left[A, B^{-1}\right]\right\|_{\alpha \rightarrow \alpha-1}\left\|R_{s}\right\|_{\alpha \rightarrow \alpha} d s, \\
& \int_{0}^{t}\left\|e^{-(t-s) A}\right\|_{\gamma-1 \rightarrow \beta}\left\|B\left[A, B^{-1}\right]\right\|_{\gamma \rightarrow \gamma-1}\left\|R_{s+t}\right\|_{\alpha \rightarrow \gamma} d s .
\end{aligned}
$$

By a process that should by now be familiar to the reader, the first integral can be estimated by $O\left(t^{1 / 2+(\alpha-\beta) / 2}\right)$ for arbitrary $\beta \geqslant \alpha-1$. In the second integral we shall use the inductive hypothesis and choose $\gamma \in[\alpha, \alpha+k$ [ (close to $\sim \alpha+k$ ) we must also have $\beta \in[\gamma-1, \gamma+1$ [ (so that $|\beta-\gamma+1|<2$ which gives convergence of the integral at the upper end!). For $\beta \in[\alpha+k, a+k+1$ [ we obtain therefore the required estimate $O\left(t^{1 / 2+(\alpha-\beta) / 2}\right)$ of that integral. We conclude that

$$
\left\|e^{-t A}-R_{t}\right\|_{\alpha \rightarrow \beta}=O\left(t^{1 / 2+(\alpha-\beta) / 2}\right)
$$

for $\beta \in\left[\alpha+k, \alpha+k+1\right.$ [. This together with the estimate $\left\|e^{-t A}\right\|_{\alpha \rightarrow \beta}=$ $O\left(t^{\alpha-\beta) / 2}\right) \beta \geqslant \alpha$ that always holds completes the inductive step for (5.4). The proof is now complete because once we know (5.4) for arbitrary $\beta \geqslant \alpha$ the estimate (5.3) for arbitrary $\beta \geqslant \alpha$ the estimate (5.3) follows by the previous argument.

At this stage I shall become more brief. The double «commutator» estimate of the form

$$
\left\|\left(c_{\lambda_{1}}-c_{\lambda_{2}}\right)\left(c_{\lambda_{3}}-c_{\lambda_{4}}\right) e^{-t A}\right\|_{\alpha \rightarrow \beta}=O\left(t^{1+(\alpha-\beta) / 2}\right)
$$

at least for $\beta \geqslant \alpha$ (with the same refinements as in Section 4 for $\beta \in[\alpha-2, \alpha]$ ) will be proved the same way as in Section 4. If anything, things are simpler 
here because the situation is more symmetric. We have for instance

$$
\left(c_{\lambda}-c_{\mu}\right)\left(c_{\nu}(P)\right)=\left(c_{\lambda+\nu}-c_{\mu+\nu}\right)(P)=c_{\nu}\left(c_{\lambda}-c_{\mu}\right)(P) .
$$

To control the «middle terms» (the analogue of $I_{2}$ of Section 4) we have to check that for $k=0,1,2, \ldots$

$$
\left\|\left(c_{\lambda}-c_{\lambda}\right)\left(c_{\lambda}-c_{\lambda}\right)\left(A^{k}\right)\right\|_{\alpha-\alpha-2 k+2} \leqslant C .
$$

The $\lambda$ 's above are of course in general different (and this a notational convention that will be adopted from here onwards). (5.11) for $\alpha=2 n$ is done again by commuting pseudo-differentials and using the singular integral estimate (4.2). We then dualize and interpolate.

Just as before the use of Section 1 of (5.11) and of formula (3.4) gives

$$
\left\|\left(c_{\lambda}-c_{\lambda}\right)\left(c_{\lambda}-c_{\lambda}\right)\left(A^{\sigma}\right)\right\|_{\alpha \rightarrow \alpha-2 \operatorname{Re} \sigma+2} \leqslant C
$$

provided that $\operatorname{Re} \sigma<0$.

We shall then develop

$$
\left(c_{\lambda}-c_{\lambda}\right)\left(c_{\lambda}-c_{\lambda}\right)\left(A^{k+\sigma}\right)
$$

for $k=1,2, \ldots \operatorname{Re} \sigma<0$ and combine (5.11), (5.2) and (5.12) (for $\operatorname{Re} \sigma<0$ ) to conclude that (5.12) holds in general for any $\sigma \in \mathbb{C}$.

Any multiple difference can be treated that way and we obtain

$$
\left\|\left(c_{\lambda}-c_{\lambda}\right)^{k}\left(A^{\sigma}\right)\right\|_{\alpha \rightarrow \beta}=\left\|\left(c_{\lambda}-c_{\lambda}\right) \cdots\left(c_{\lambda}-c_{\lambda}\right)\left(A^{\sigma}\right)\right\|_{\alpha \rightarrow \beta} \leqslant C
$$

for $\beta=\alpha-2 \operatorname{Re} \sigma+k$. This will not be used for our main application in this paper for any $k>2$.

We shall finally need to control mixed expressions of the form

$$
M_{1}=\left(I-c_{\lambda}\right)\left[A^{\sigma}, E\right], \quad M_{2}=\left[\left(I-c_{\lambda}\right)\left(A^{\sigma}\right), E\right]
$$

and of course multiple commutators with $E \in S_{1,0}^{0}$ and powers of the operation $\left(c_{\lambda}-c_{\lambda}\right)$ can also be considered.

It should be clear to the reader by now what the appropriate estimate is. Just for the record, let me sketch a proof of the fact that

$$
\left\|M_{1}\right\|_{\alpha \rightarrow \beta}, \quad\left\|M_{2}\right\|_{\alpha \rightarrow \beta} \leqslant C
$$

for $\beta=\alpha-2 \operatorname{Re} \sigma+2$. Observe also that

$$
\left\|M_{1}-M_{2}\right\|_{\alpha \rightarrow \beta}=\left\|B^{-1}[B, E] A^{\sigma}\right\|_{\alpha \rightarrow \beta}+\left\|\left[B^{-1}, E\right]\left[B, A^{\sigma}\right]\right\|_{\alpha \rightarrow \beta}
$$

(for any operator norm), where $B=\Lambda^{s}$. We shall see in the next section that we can control the terms on the right since they are of the form $\left\|S_{1} A^{\sigma}\right\|$ and 
$\left\|S_{2}\left[B, A^{\sigma}\right]\right\|$ for appropriate pseudo-differential operators $S_{i}$. We can therefore pass early from $M_{1}$ to $M_{2}$ and vice versa, (at least this is the case when $\beta=0$ and, in a final analysis, as we shall see, this is all that counts). At any rate, we shall not need this passage from $M_{1}$ to $M_{2}$ here since we can estimate $M_{1}$ and $M_{2}$ separately just as easily in a symmetrical manner.

Indeed observe first that

$$
c_{\lambda}\left(\left[T_{t}, E\right]\right)=\left[c_{\lambda}\left(T_{t}\right), E_{\lambda}\right]
$$

with $E \rightarrow E_{\lambda}=c_{\lambda}(E)$ a bijection on $S_{1,0}^{0}$. It therefore is enough to control one of the two terms (5.13). Say

$$
\begin{aligned}
c_{\lambda}\left(\left[T_{t}, E\right]\right)= & \int_{0}^{t} c_{\lambda}\left(e^{-(2 t-s) A}\right) c_{\lambda}([A, E]) c_{\lambda}\left(e^{-s A}\right) d s \\
& +\int_{0}^{t} c_{\lambda}\left(e^{-(t-s) A}\right) c_{\lambda}([A, E]) c_{\lambda}\left(e^{-(s+t) A}\right) d s .
\end{aligned}
$$

Bearing in mind the estimate (5.4) and the following estimate

$$
\left\|c_{\lambda}[A, E]\right\|_{\alpha \rightarrow \alpha-1}=\left\|\left[c_{\lambda}(A), E_{\lambda}\right]\right\|_{\alpha \rightarrow \alpha-1} \leqslant C
$$

valid for all $\alpha \in \mathbb{R}$ we obtain as before

$$
\text { (5.15) }\left\|c_{\lambda}\left[T_{t}, E\right]\right\|_{\alpha \rightarrow \beta}, \quad\left\|\left[c_{\lambda}\left(T_{t}\right), E\right]\right\|_{\alpha \rightarrow \beta}=O\left(t^{1 / 2+(\alpha-\beta) / 2}\right), \quad \beta \geqslant \alpha-1 .
$$

The estimate (5.14) can (probably!) be reduced to the other similar estimates that we have already examined. There is no need to do so however; a direct proof along the same lines as our previous similar proofs is preferable. Indeed (5.14) is clear by pseudo-differential calculus and the sub-elliptic estimate (4.2) (just as in the proof of (5.11)) when $\alpha=2 k+1 \quad(k=1,2, \ldots)$. We simply dualize and interpolate as before.

We next write (we use our convention in (5.11) for the various $c_{\lambda}$ 's)

$$
\begin{aligned}
{\left[\left(c_{\lambda}-c_{\lambda}\right) e^{-2 t A}, E\right]=} & {\left[\int_{0}^{t} c_{\lambda}\left(e^{-(2 t-s) A}\right)\left(c_{\lambda}-c_{\lambda}\right)(A) c_{\lambda}\left(e^{-s A}\right) d s, E\right] } \\
& +\left[\int_{0}^{t} c_{\lambda}\left(e^{-(t-s) A}\right)\left(c_{\lambda}-c_{\lambda}\right)(A) c_{\lambda}\left(e^{-(s+t) A}\right) d s, E\right]
\end{aligned}
$$

and we see from (5.15) that we can subject this expression to the same treatment as in the proof of $\left[\left[e^{-2 t A}, E_{1}\right], E_{2}\right]$ or $\left(c_{\lambda}-c_{\lambda}\right)\left(c_{\lambda}-c_{\lambda}\right) e^{-2 t A}$ as soon as we have the estimate

$$
\left\|\left[\left(c_{\lambda}-c_{\lambda}\right) A, E\right]\right\|_{\alpha \rightarrow \alpha} \leqslant C .
$$

We have of course more generally

$$
\left\|\left[\left(c_{\lambda}-c_{\lambda}\right) A^{k}, E\right]\right\|_{\alpha \rightarrow \beta},\left\|\left(c_{\lambda}-c_{\lambda}\right)\left[A^{k}, E\right]\right\|_{\alpha \rightarrow \beta} \leqslant C,
$$


with $\beta=\alpha+2 k-2$ with the same proofs as before (i.e. we first prove it for $\alpha=2 n$, an even integer).

The estimate

$$
\left\|\left[\left(c_{\lambda}-c_{\lambda}\right) A^{\sigma}, E\right]\right\|_{\alpha \rightarrow \beta} \leqslant C
$$

for $\beta=\alpha+2 \operatorname{Re} \sigma-2$ follows by the same machinery. Notice that when going through this last step of that proof we must use the fact that the $\|\bullet\|_{\alpha \rightarrow \alpha-2 \operatorname{Re} \rho+1}$ norm of $c_{\lambda}\left[A^{\rho}, E\right]=\left[c_{\lambda}\left(A^{\rho}\right), \tilde{E}\right]$ is bounded, for $\rho=k=$ $1,2, \ldots$ or $\operatorname{Re} \rho<0$. But this is clear from the corresponding estimate

$$
\left\|\left[\left(I-c_{\lambda}\right)\left(A^{\rho}\right), E\right]\right\|_{\alpha \rightarrow \alpha-2 \operatorname{Re} \rho+2} \leqslant C
$$

and the already known estimate of $\left\|\left[A^{\rho}, E\right]\right\|_{\alpha \rightarrow \alpha-2 \operatorname{Re} \rho+1}$.

The estimate for $M_{1}$ is identical and I will spare the reader the details.

\section{The Classical Sobolev Spaces $H_{\alpha}$}

In this section I shall face some new aspects of the theory. First of all, I shall change the scale and consider the scale of classical Sobolev spaces

$$
H_{\alpha}=\left\{f: \Lambda^{\alpha} f \in X\right\}
$$

where $X=L^{2}\left(\mathbb{R}^{n}\right)$ throughout, and $\Lambda=(I+\Delta)^{1 / 2}$ for the standard Laplacian $\Delta$. I shall refer to $\|\cdot\|_{\alpha}=\|\cdot\|_{H_{\alpha}},\|\cdot\|_{\alpha \rightarrow \beta}=\|\cdot\|_{H_{\alpha} \rightarrow H_{\beta}}$ and it is important of course that no confusion should arise with the norms $\|\cdot\|_{X_{\alpha} \rightarrow X_{\beta}}$ considered in the previous two sections. In this section we shall analyse the mapping properties of operators of the form $P=\left[A^{\sigma}, E\right], P=\left(c_{\lambda}-c_{\lambda}\right)\left(A^{\sigma}\right) \cdots$ of Section 4, Section 5 (and even more general operators) between spaces of this classical Sobolev scale i.e. $P: H_{\alpha} \rightarrow H_{\beta}$.

The strange thing is that to do so we shall use the results of the previous two sections but only when $\beta=0$ i.e. from the previous two sections we shall only retain information of the form

$$
\left\|\left[A^{\sigma}, E\right] f\right\|_{X} \leqslant C\left\|A^{\sigma-1 / 2} f\right\|_{X}
$$

or more generally $\|P f\|_{X} \leqslant C\left\|A^{t} f\right\|_{X}$ for an appropriate $t \in \mathbb{R}$. Our aim consists simply of generalizing these estimates to the $H_{\alpha}(\alpha \in \mathbb{R})$ scale so that one of the things that we shall see, for instance, is

$$
\left\|\left[A^{\sigma}, E\right]\right\|_{H_{\alpha}} \leqslant C\left\|A^{\sigma-1 / 2} f\right\|_{H_{\alpha}},
$$

for all $\alpha \in \mathbb{R}$.

In the considerations that follows the letter $B$ will be reserved for some power $B=\Lambda^{s}(s \in \mathbb{R})$. 
The first thing to prove is that we have $\left\|B A^{\sigma} B^{-1} f\right\|_{X} \approx\left\|A^{\sigma} f\right\|_{X}$ in the sense that

$$
C^{-1}\left\|A^{\sigma} f\right\|_{X} \leqslant\left\|B A^{\sigma} B^{-1} f\right\|_{X} \leqslant C\left\|A^{\sigma} f\right\|_{X}
$$

for $\sigma \in \mathbb{C}$ and some $C>0$ independent of $f$.

One of the two inequalities is evident. Indeed

$$
\begin{aligned}
\left\|B A^{\sigma} B^{-1} f\right\|_{X} & \leqslant C\left\|A^{\sigma} f\right\|_{X}+C\left\|B\left[A^{\sigma}, B^{-1}\right] f\right\|_{X} \\
& \leqslant C\left(\left\|A^{\sigma} f\right\|_{X}+\left\|A^{\sigma-1 / 2} f\right\|_{X}\right) \\
& \leqslant C\left\|A^{\sigma} f\right\|_{X}
\end{aligned}
$$

by Section 5 (and the fact that $\left\|A^{\sigma+j} f\right\|_{X} \geqslant C\left\|A^{\sigma} f\right\|_{X}, j \geqslant 0$ ).

For the other way round we shall assume first that $\operatorname{Re} \sigma>0$ and just as before, because of Section 5, observe that

$$
\begin{aligned}
\left\|A^{\sigma} f\right\|_{X} & \leqslant C\left[\left\|B A^{\sigma} B^{-1} f\right\|_{X}+\left\|A^{\sigma-1 / 2} f\right\|_{X}\right] \\
& \leqslant C \sum_{j=0}^{k-1}\left\|B A^{\sigma-j / 2} B^{-1}\right\|_{X}+C\left\|A^{\sigma-k / 2} f\right\|_{X}
\end{aligned}
$$

for any $k=0,1, \ldots$. If we choose a $k$ large enough for $\operatorname{Re}(\sigma-k / 2)<0$ we have

$$
\left\|A^{\sigma-k / 2} f\right\|_{X} \leqslant C\|f\|_{X} \leqslant C\left\|B A^{\sigma} B^{-1} f\right\|_{X} .
$$

The last inequality holds because $\operatorname{Re} \sigma>0$ and because $e^{-t A}$ acts on $H_{s}$. Indeed our convention ( $c f$. Section 1 ) about the behaviour at infinity of that semigroup guarantees that

$$
B A^{\rho} B^{-1}: X \rightarrow X
$$

is bounded if $\operatorname{Re} \rho<0$. Since the terms under the summation sign of (6.2) are decreasing in $j$ we finally obtain that $\left\|A^{\sigma} f\right\|_{X} \approx\left\|B^{-1} A^{\sigma} B\right\|_{X}$ as long as $\operatorname{Re} \sigma>0$. By taking inverses this of course means that the same is true for $\operatorname{Re} \sigma<0$. The pure imaginary $\sigma$ 's are evident since $A^{i t}(t \in \mathbb{R})$ act boundedly on $H_{\alpha}$.

The above has several consequences. In particular, we see that the norms

$$
\|f\|_{a, \sigma}=\left\|\Lambda^{\alpha} A^{\sigma} \Lambda^{\beta} f\right\|_{X} \quad \text { where } \quad \alpha+\beta=a \text { is fixed }
$$

are all equivalent norms for fixed $a \in \mathbb{R}, \sigma \in \mathbb{C}$. (To see this one applies (6.1) with $f=\Lambda^{\gamma} \phi$ for appropriate $\gamma$.)

The next thing to observe is that

$$
\left\|\left[A^{\sigma}, E\right] f\right\|_{m} \leqslant\left\|A^{\sigma-1 / 2} f\right\|_{m}
$$


$\left(\sigma \in \mathbb{C}, m \in \mathbb{R}\right.$ ). Indeed, perform the change of variables $f=\Lambda^{-m} \phi$ and the above inequality is simply transformed (because of (6.1)) into

$$
\left\|c_{m}\left[A^{\sigma}, E\right] \phi\right\|_{X} \leqslant C\left\|A^{\sigma-1 / 2} \phi\right\|_{X} .
$$

This is a special case of (5.16) in Section 5 (and the few lines that follow).

Let us now consider the most general inequality of this type with one or less commutators, this is

$$
\left\|S^{n_{1}} A^{\sigma} S^{n_{2}}\right\|_{m} \leqslant\left\|A^{\sigma} f\right\|_{p}, \quad\left\|S^{n_{1}}\left[A^{\sigma}, S^{n_{2}}\right] S^{n_{2}} f\right\|_{m} \leqslant C\left\|A^{\sigma-1 / 2} f\right\|_{p}
$$

where $S^{n_{i}} \in O P S_{1,0}^{n_{i}}$ and where we suppose that $\sum n_{i}+m=p$. I shall prove the second, the first being easier. I proceed as follows: I first factor $S^{n_{i}}=\Lambda^{n_{i}} E$ $=E \Lambda^{n_{i}}$, expand the $\left[{ }^{\circ}, \cdot\right]$ and refactor, if necessary, to bring it to the form

$$
\left\|E \Lambda^{\nu_{1}}\left[A^{\sigma}, P\right] \Lambda^{\nu_{2}} E f\right\|_{X} \leqslant C\left\|A^{\sigma-1 / 2} f\right\|_{p}
$$

with $P=E$ or $\Lambda^{\nu}$ and ord $P+\nu_{1}+\nu_{2}=p$ (here we set $\operatorname{ord} E=0$, ord $\Lambda^{\nu}=\nu$ ). The two $E$ 's can be eliminated. The one on the left is absorbed in the $\|\cdot\|_{X}$, the one on the right disappears by the change of variable $E f=\phi$ and (6.4). A final change of variable $\psi=\Lambda^{-p} \phi$ reduces everything to the case $p=0$ (we use (6.1) for that). So we are finally left with proving

$$
\left\|\Lambda^{\mu_{1}}\left[A^{\sigma}, P\right] \Lambda^{\mu_{2}} f\right\|_{X} \leqslant C\left\|A^{\sigma-1 / 2} f\right\|_{X}
$$

with $\mu_{1}+\mu_{2}+\operatorname{ord} P=0$. But here we are on familiar ground, for the operator on the left is in one of the following two forms:

$$
\left(c_{\lambda}-c_{\lambda}\right)\left(A^{\sigma}\right), \quad c_{\lambda}\left[A^{\sigma}, E\right] .
$$

Both of these have been dealt with in Sections 4 and 5.

Let us now pass on to second commutators. The estimate we shall need is the following

$$
\left\|S^{n_{1}}\left[\left[A^{\sigma}, S^{n_{2}}\right], S^{n_{3}}\right] S^{n_{4}} f\right\|_{m} \leqslant C\left\|A^{\sigma-1} f\right\|_{p}
$$

where $p=m+\sum n_{i}$, and $S^{n_{i}} \in O P S_{1,0}^{n_{i}}$. The proof is easy. Use the factorization of $S^{n} \in O P S_{1,0}^{n}$ into $S^{n}=\Lambda^{n} E=E \Lambda^{n}$ a number of times over and we see that the expression on the left can be estimated by

$$
\left\|E \Lambda^{\nu_{1}}\left[A^{\sigma}, R\right] \Lambda^{\nu_{2}} E f\right\|_{X}+\left\|E \Lambda^{\mu_{1}}\left[\left[A^{\sigma}, P\right], Q\right] \Lambda^{\mu_{2}} E f\right\|_{X}
$$

$P, Q, R=E$ or $\Lambda^{s}$ for some $s \in \mathbb{R}$.

Just as before, the two $E$ 's on the above two expressions can be disregarded. The one on the left is absorbed in the norm $\|\cdot\|_{X}$ the one on the right disappears by the change of variables $\phi=E f$ since we clearly have, from what has already been done, $\left\|A^{\sigma-1} \phi\right\|_{p} \leqslant C\left\|A^{\sigma-1} f\right\|_{p}$. The first term in (6.5) has already been 
treated and turns out to be bounded by $\left\|A^{\sigma-1 / 2} f\right\|_{p-1}$. To check that it is indeed the $H_{p-1}$ norm that we actually have here, we must consider cases. The idea, however, is simple: since only one commutator appears in this term this means that two pseudo-differentials have been «contracted» by a commutation $\left[S^{r_{1}}, S^{r_{2}}\right]$ but that is of course a pseudo-differential that belongs to $O P S_{1,0}^{r_{1}+r_{2}-1}$ and that explaining the gain of one derivative that gives $p-1$. Since, by Section $2, A^{1 / 2}: H_{p} \rightarrow H_{p-1}$ is bounded, we see that $\left\|A^{\sigma-1 / 2} f\right\|_{p-1} \leqslant$ $\left\|A^{\sigma-1} f\right\|_{p}$. We thus see that the first term in (6.5) is controlled.

To control the second term in (6.5) we start by the change of variable $f=\Lambda^{-p} \phi$ and a use of (6.1) that reduces the problem to showing

$$
\left.\| \Lambda^{\nu}\left[A^{\sigma}, P\right], Q\right] \Lambda^{\mu} f\left\|_{X} \leqslant C\right\| A^{\sigma-1} f \|_{X}
$$

with $P, Q=\Lambda^{\lambda}$ or $E$ and $\operatorname{ord} P+\operatorname{ord} Q+\nu+\mu=0$ (here again ord $E=0$, ord $\Lambda^{\nu}=\nu$ ). This is a familiar expressions from Section 4 and 5. The four cases $P, Q=E, \Lambda^{\lambda}$ have to be checked separately

(1) $P, Q=\Lambda^{\lambda}$ gives

$$
\Lambda^{\nu}\left[\left[A^{\sigma}, P\right], Q\right] \Lambda^{\mu}=\left(c_{\lambda}-c_{\lambda}\right)\left(c_{\lambda}-c_{\lambda}\right) A^{\sigma} .
$$

(2) $P, Q=E$ gives us a term of the form $\left[\left[c_{\lambda}\left(A^{\sigma}\right), E_{1}\right], E_{2}\right]$ which can be controlled by the three terms $\left[\left[A^{\sigma}, E_{1}\right], E_{2}\right], E_{2}\left[\left(I-c_{\lambda}\right) A^{\sigma}, E_{1}\right]$ and $\left[\left(I-c_{\lambda}\right) A^{\sigma}, E_{1}\right] E_{2}$. The $E_{2}$ on the right of the last term is then eliminated by the change of variables $E_{2} f=\phi$.

(3) $P=E, Q=\Lambda^{\lambda}$ gives us a term of the form $\left(c_{\lambda}-c_{\lambda}\right)[A, E]$.

(4) $P=\Lambda^{\lambda}, Q=E$ is reduced to the previous case modulo $\Lambda^{\nu}\left[A^{\sigma}, S^{\lambda-1}\right] \Lambda^{\mu}$ with $S^{\lambda-1} \in O P S_{1,0}^{\lambda-1}$.

We shall go no further here. It is clear however that if we proceed that way we obtain the basic commutation estimate of the introduction (at least for «sum of squares» sub-elliptic operators). Things can be presented in an even more symmetrical way. We can denote by

$$
Z_{\beta}^{\alpha}=\left\{f: A^{\alpha / 2} \Lambda^{\beta} f \in X\right\}
$$

which is a double index scale of spaces. The order at which $A$ and $\Lambda$ are set is immaterial ( $c f .(6.3)$ ). From the above considerations together with some additional work ( $c f$. Section 11) we can establish the precise mapping properties in $Z_{\beta}^{\alpha}$ of commutators of one power of $A$ with an arbitrary number of pseudodifferentials. I shall not pursue the matter further here. A typical result that can thus be obtained is

$$
\left\|A^{t}\left[A^{\sigma}, S^{n}\right] f\right\|_{H_{\alpha}} \leqslant C\left\|A^{t+s-1 / 2} f\right\|_{H_{\alpha+n}}, \quad S^{n} \in O P S_{1,0}^{n} .
$$




\section{The Interaction of Two Distinct Operators}

We shall now consider $L$ and $M$, two distinct differential operators, both of which I shall assume are sums of squares of fields

$$
L, M=\sum X_{j}^{*} X_{j} .
$$

I shall also assume that they are both sub-elliptic

$$
\begin{aligned}
\|f\|_{1-\delta} & \leqslant C\left\|(1+L)^{1 / 2}\right\|_{X}, \\
\|f\|_{1-d} & \leqslant C\left\|(1+M)^{1 / 2}\right\|_{X}
\end{aligned}
$$

with $0 \leqslant d, \delta<1 .\left(X=L^{2}\right)$. The spaces $H_{\alpha}$ are as before the standard Sobolev spaces of Section 6 and $\|\cdot\|_{\alpha}=\|\cdot\|_{H_{\alpha}}$ refer to these norms. The aim of this section is to prove the following basic

Proposition. Let $L, M, \delta$ and $d$ be as above and assume that $d+\delta \leqslant 1$. The semigroup $e^{-t M}$ acts as a continuous semigroup on each space $X_{\alpha}(\alpha \in \mathbb{R})$ induced by the operator $L$ (cf. (Section 4$)$ ).

In fact a slightly more general result will be proved (under the same assumption that $\delta+d \leqslant 1$ ). I shall prove that for all $\beta \geqslant \alpha$ and all $j \in \mathbb{R}$ we have

$$
\left\|(I+L)^{j / 2} e^{-t M}(1+L)^{-j / 2}\right\|_{\alpha \rightarrow \beta}=O\left(t^{(\alpha-\beta) / 2(1-d)}\right) .
$$

For $\alpha=\beta=0$ this is the content of our proposition. Observe straight away that it suffices to prove for $j=0,1, \ldots$. Indeed the action of $(I+L)^{i s}(s \in \mathbb{R})$ on $H_{\alpha}$ implies that we then have the same result for $j \in \mathbb{C}, \operatorname{Re} j=0,1, \ldots$. Complex interpolation of an analytic family of operators for fixed $\alpha$ and $\beta$ gives then (7.2) for any $j \in \mathbb{C}, \operatorname{Re} j>0$ we then dualize and have the general result. (In the above complex interpolation there is a slight difficulty: We have to recover the factor $t^{(\alpha-\beta) / 2(1-d)}$ for the interpolated operator. This is no problem here since we have the same factor at both ends of the interpolation interval $c f$. [4]. Things would have been different if we had different factors $C_{1}(t)$ and $C_{2}(t)$. It would not then be necessarily true that we obtain $C_{1}^{\theta} C_{2}^{1-\theta}$ after interpolation. At any rate, if you do not feel comfortable with complex interpolation, proceed differently: Prove (7.2) for $j=0,1, \ldots$ and general $\alpha, \beta \in \mathbb{R}, \beta \geqslant \alpha$, and then interpolate only when $\alpha=\beta=0$. We still obtain our proposition.)

The fact that (7.2) holds for $j=0$ is just the content of (2.5). We shall assume therefore that (7.2) holds for $j=0,1, \ldots, k$, and prove it for $k+1$. The remainder of this section will be devoted to this task. 
Our first step will be to analyse the expression

$$
\begin{aligned}
{\left[M,(1+L)^{(k+1) / 2}\right] } & =\sum_{j}\left[X_{j}^{*} X_{j},(1+L)^{(k+1) / 2}\right] \\
& =\sum_{j} X_{j}^{*}\left[X_{j},(I+L)^{(k+1) / 2}\right]+\sum_{j}\left[X_{j}^{*},(1+L)^{(k+1) / 2}\right] X_{j},
\end{aligned}
$$

$X_{j}$ will be, of course, the fields $X_{j}$ in the decomposition of $M$ and the index $j$ as well as the summation will be dropped from here onwards. We shall rewrite this in the following form

$$
\begin{array}{r}
M^{1 / 2}\left\{M^{-1 / 2} X^{*}\left[X,(I+L)^{(k+1) / 2}\right]+M^{-1 / 2} X\left[X^{*},(I+L)^{(k+1) / 2}\right]\right\} \\
+\left[\left[X^{*},(I+L)^{(k+1) / 2}\right], X\right] .
\end{array}
$$

For all the $X^{\prime}$ s or $X^{*}$ 's that are inside a bracket $\left[\cdot, \cdot{ }^{\circ}\right.$ the only information that we shall retain is the fact that they belong to $O P S_{1,0}^{1}$. Then we can use the results of our previous sections to estimate all the brackets that appear in (7.3). The double bracket $B_{2}$ that appears at the end of (7.3) satisfies for instance $\left\|B_{2} f\right\|_{\alpha} \leqslant C\left\|(1+L)^{(k-1) / 2} f\right\|_{\alpha+2}(\alpha \in \mathbb{R})$ and the single brackets $B_{1}$ that appear within $\{\bullet\}$ in (7.3) satisfy

$$
\left\|B_{1} f\right\|_{\alpha} \leqslant C\left\|(I+L)^{k / 2} f\right\|_{\alpha+1} .
$$

The two «Riesz transforms» satisfy

$$
\left\|M^{-1 / 2} X^{*} f\right\|_{\alpha}, \quad\left\|M^{-1 / 2} X f\right\|_{\alpha} \leqslant C\|f\|_{\alpha} .
$$

The $X^{*}$ can in fact be ignored in (7.4) since $X-X^{*} \in S_{1,0}^{0}$. The estimate (7.4) holds in general even without sub-ellipticity, i.e. $d=1$. To see this, considering the adjoint operator, it is clearly enough to show that

$$
\|X f\|_{\alpha} \leqslant C\left\|M^{1 / 2} f\right\|_{\alpha} \quad(\alpha \in \mathbb{R})
$$

(by our convention $M$ is really $\lambda_{0}+M, \lambda_{0}>0$ ). This last estimate is clear enough since

$$
\begin{aligned}
\|X f\|_{\alpha} & =\left\|\Lambda^{\alpha} X f\right\|_{X} \\
& \leqslant C\left(\left\|X \Lambda^{\alpha} f\right\|_{X}+\|f\|_{\alpha}\right) \\
& \leqslant C\left\|M^{1 / 2} \Lambda^{\alpha} f\right\|_{X}+C\|f\|_{\alpha} \\
& \leqslant C\left(\left\|M^{1 / 2} f\right\|_{\alpha}+C\left\|\left[M^{1 / 2}, \Lambda^{\alpha}\right] f\right\|_{0}\right. \\
& \leqslant C\left\|M^{1 / 2} f\right\|_{\alpha}
\end{aligned}
$$

(since $M^{-1 / 2}: H_{\alpha} \rightarrow H_{\alpha}$ is bounded) by the results of Section 6 . In fact, a closer analysis of the above proof shows that the «M. Riesz transfer theorem» works both ways on the $H_{\alpha}$ scale (even without sub-ellipticity conditions) 


$$
\begin{aligned}
C^{-1}\left\|M^{1 / 2} f\right\|_{\alpha} & \leqslant \sum_{j}\left\|X_{j} f\right\|_{\alpha}+\|f\|_{\alpha} \\
& \leqslant C\left\|M^{1 / 2} f\right\|_{\alpha} .
\end{aligned}
$$

The analogue for higher Riesz transforms also holds but it does need subellipticity and is harder to prove ( $c f$. Section 11)

$$
X_{i_{1}} \cdots X_{i_{k}} M^{-k / 2}: H_{\alpha} \rightarrow H_{\alpha}, \quad \alpha \in \mathbb{R}, \quad k=1,2, \ldots
$$

is a bounded operator.

At any rate we shall not need here these higher Riesz transforms. What we need rather is to combine all the above information and simply conclude that

$$
\left[M,(I+L)^{(k+1) / 2}\right]=M^{1 / 2} A+B
$$

where

$$
A(1+L)^{-k / 2}: H_{\alpha+1} \rightarrow H_{\alpha}, \quad B(1+L)^{-(k-1) / 2}: H_{\alpha+2} \rightarrow H_{\alpha}
$$

are bounded operators $(k=1,2, \ldots, \alpha \in \mathbb{R})$.

We shall now denote by

$$
R_{t}^{(k)}=(1+L)^{k / 2} e^{-t M}(1+L)^{-k / 2}
$$

and analyse the expression

$$
\left[e^{-2 t M},(1+L)^{(k+1) / 2}\right](I+L)^{-(k+1) / 2}=e^{-2 t M}-R_{2 t}^{(k+1)}=I(t)
$$

we first decompose

$\left[e^{-2 t M},(I+L)^{(k+1) / 2}\right]=e^{-t M}\left[e^{-t M},(1+L)^{(k+1) / 2}\right]+\left[e^{-t M},(I+L)^{(k+1) / 2}\right] e^{-t M}$

and then write

$$
\begin{aligned}
I(t)= & \int_{0}^{t} e^{-(2 t-s) M}\left[M,(1+L)^{(k+1) / 2}\right] e^{-s M} d s(1+L)^{-(k+1) / 2} \\
& +\int_{0}^{t} e^{-(t-s) M}\left[M,(1+L)^{(k+1) / 2}\right] e^{-(t+s) M} d s(1+L)^{-(k+1) / 2} .
\end{aligned}
$$

In what follows I shall assume that $k \geqslant 1$ (the case $k=0$ is much easier but has to be treated separately). The middle term in the two integrals above will be decomposed as in (7.5) and we shall have to deal separately with four different terms $I_{i}(t), i=1,2,3,4$. We have

$$
\left\|I_{1}(t)\right\|_{\alpha \rightarrow \beta} \leqslant \int_{0}^{t}\left\|e^{-(2 t-s) M} M^{1 / 2}\right\|\left\|A(I+L)^{-(k / 2)}\right\|\left\|R_{s}^{(k)}\right\| d s\left\|(1+L)^{-1 / 2}\right\|
$$


where we have a «cascade» of \|\|$_{s \rightarrow t}$ norms that run as follows

$$
\begin{aligned}
\left\|(I+L)^{-1 / 2}\right\|_{\alpha \rightarrow \alpha+1-\delta} & \leqslant C, \\
\left\|A(I+L)^{-k / 2}\right\|_{\alpha+1-\delta \rightarrow \alpha-\delta} & \leqslant C, \\
\left\|R_{s}^{(k)}\right\|_{\alpha+1-\delta \rightarrow \alpha+1-\delta} & =O(1), \\
\left\|e^{-(2 t-s) M_{M}} M^{1 / 2}\right\|_{\alpha-\delta \rightarrow \beta} & =O\left((2 t-s)^{(\alpha-\beta-\delta) / 2(1-d)-1 / 2}\right) .
\end{aligned}
$$

The first of these estimates is a consequence of (2.6). The third estimate is of course our introduction hypothesis, the second is (7.6). To obtain the fourth, we use the holomorphicity of the action of the semigroup $e^{-t M}$ on each $H_{\alpha}$ space that allows us to conclude together with (2.6) that

$$
\left\|M^{\lambda} e^{-2 t M}\right\|_{r \rightarrow s}=\left\|M^{\lambda} e^{-t M}\right\|_{s \rightarrow s}\left\|e^{-t M}\right\|_{r \rightarrow s}=O\left(t^{(r-s) / 2(1-d)-\lambda}\right)
$$

for all $s \geqslant r, \lambda \geqslant 0$. If we put all the above estimates together and integrate we obtain that

$$
I_{1}(t)=O\left(t^{(\alpha-\beta) / 2(1-d)+(1-\delta-d) / 2(1-d)}\right) .
$$

The $I_{3}$ term is quite analogous except that the $(2 t-s)$ has to be replaced by $(t-s)$ and the $s$ in $R_{s}^{(k)}$ has to be replaced by $t+s$ and we shall perform the jump form $\alpha$ to $\beta$ at the first level. We have again for the same reasons

$$
\begin{aligned}
\left\|(I+L)^{-1 / 2}\right\|_{\alpha \rightarrow \alpha+1-\delta} & \leqslant C, \\
\left\|R_{s+t}^{(k)}\right\|_{\alpha+1-\delta \rightarrow \beta+1} & =O\left((t+s)^{(\alpha-\beta-\delta) / 2(1-d)}\right), \\
\left\|A(I+L)^{-k / 2}\right\|_{\beta+1 \rightarrow \beta} & \leqslant C, \\
\left\|e^{-(t-s) M} M^{1 / 2}\right\|_{\beta \rightarrow \beta} & =O\left((t-s)^{-1 / 2}\right),
\end{aligned}
$$

after integration we obtain exactly the same estimate for $I_{3}(t)$.

The decomposition of $I_{2}(t)$ is different

$$
\left\|I_{2}(t)\right\|_{\alpha \rightarrow \beta} \leqslant \int_{0}^{t}\left\|e^{-(2 t-s) M}\right\|\left\|B(I+L)^{(-k+1) / 2}\right\|\left\|R_{s}^{(k-1)}\right\| d s\left\|(1+L)^{-1}\right\| .
$$

The estimates are similar and for the same reasons

$$
\begin{aligned}
\left\|(I+L)^{-1}\right\|_{\alpha \rightarrow \alpha+2-2 \delta} & \leqslant C \\
\left\|B(I+L)^{-(k-1) / 2}\right\|_{\alpha+2-2 \delta \rightarrow \alpha-2 \delta} & \leqslant C \\
\left\|R_{s}^{(k-1)}\right\|_{\alpha+2-2 \delta \rightarrow \alpha+2-2 \delta} & =O(1) \\
\left\|e^{-(2 t-s) M}\right\|_{\alpha-2 \delta \rightarrow \beta} & =O\left((2 t-s)^{(\alpha-\beta-2 \delta) / 2(1-d)}\right) .
\end{aligned}
$$

After integration we obtain the estimate $I_{2}(t)=O\left(t^{(\alpha-\beta) / 2(1-d)+(1-\delta-d) /(1-d)}\right)$. The final term $I_{4}(t)$ is of course like the $I_{2}$ with: $2 t-s \leftrightarrows t-s, s \rightleftarrows t+s$ interchanged, and the jump performed at the first level 


$$
\begin{aligned}
\left\|(I+L)^{-1}\right\|_{\alpha \rightarrow \alpha+2-2 \delta} & \leqslant C, \\
\left\|B(I+L)^{-(k-1) / 2}\right\|_{\beta+2 \rightarrow \beta} & \leqslant C, \\
\left\|R_{s+t}^{(k-1)}\right\|_{\alpha+2-2 \delta \rightarrow \beta+2} & =O\left((t+s)^{(\alpha-\beta-2 \delta) / 2(1-d)}\right), \\
\left\|e^{-(t-s) M}\right\|_{\beta \rightarrow \beta} & =O(1) .
\end{aligned}
$$

Integration gives the same estimate as $I_{2}(t)$.

We can finally complete the inductive step. Indeed, by our hypothesis: $d+\delta \leqslant 1 ; I(t)=e^{-t M}-R_{t}^{(k+1)}$ has just been seen to satisfy $\|I(t)\|_{\alpha \rightarrow \beta}=$ $O\left(t^{(\alpha-\beta) / 2(1-d)}\right)$ for $\beta \geqslant \alpha$. But since the same estimate holds for $e^{-t M}$ by (2.5). We obtain that $\left\|R_{t}^{(k+1)}\right\|_{\alpha \rightarrow \beta}=O\left(t^{(\alpha-\beta) / 2(1-d)}\right)$ which is our inductive step and we are done when $k \geqslant 1$.

Let us now examine the case $k=0$. The commutator $\left[M,(I+L)^{1 / 2}\right]$ is then factored (with the same notations as (7.3)) as follows

$$
M^{1 / 2}\left\{\left(M^{-1 / 2} X^{*}\right)\left[X,(1+L)^{1 / 2}\right]\right\}+\left\{\left[X^{*},(1+L)^{1 / 2}\right]\left(X M^{-1 / 2}\right)\right\} M^{1 / 2} .
$$

The terms within $\{\cdot\}$ have a bounded $\|\cdot\|_{\alpha+1 \rightarrow \alpha}$ norm for reasons that have been explained many times over. The $M^{1 / 2}$ on the extreme left of (7.7) once put in the integral is absorbed in the $e^{-(2 t-s) M}$ or $e^{-(t-s) M}$ and the integral converges. Similarly the $M^{1 / 2}$ on the extreme right is absorbed in the $e^{-s M}$ or $e^{-(t+s) M}$ and again gives a convergent integral. This simply gives after the computations

$$
\left\|e^{-t M}-R_{t}^{(1)}\right\|_{\alpha \rightarrow \beta}=O\left(t^{(\alpha-\beta) / 2(1-d)+(1-\delta-d) / 2(1-d)}\right)
$$

as before. In fact, the distinction of cases $k=0, k \geqslant 1$ is artificial and with a little more work I could easily have unified the presentation.

In the above proof we have used both the sub-ellipticity of $L$ and of $M$ (i.e. that both $d$ and $\delta \leqslant 1$ ). It is clear however and much easier to prove that when $\delta=0$ then $d$ could be 1 . This is just the fact that $e^{-t M}$ acts on the classical Sobolev spaces. Slightly more can be proved when $d=0$. Indeed we shall show in Section 10 that if we introduce the following scalar product on $X_{2 n}$, $n=1,2, \ldots$

$$
\ll f, f \gg_{n}=\sum_{j=1}^{n} c_{j}\left\langle(I+L)^{2 j} f, f\right\rangle_{L^{2}}
$$

then for an appropriate choice of the $c_{j} \geqslant 0$ the operator $\Delta=-\sum\left(\partial^{2} / \partial x_{i}^{2}\right)$ (the "positive» Laplacian) (or more generally any second order elliptic operator $\tilde{\Delta}$ ) is semibounded bellow

$$
\operatorname{Re} \ll \tilde{\Delta} f, f \gg_{n} \geqslant-C \ll f, f \gg_{n} .
$$

The reason why this is important is that it easily implies that $\Delta^{\mu}$ is also semibounded (by subordination) and so is, therefore, every operator of the form 
$L^{\nu}+\Delta^{\mu}(\nu \geqslant 0,0 \leqslant \mu \leqslant 1)$. As a result it follows that the semigroup $\exp \left(-t\left(L^{\nu}+\Delta^{\mu}\right)\right)$ acts as a continuous semigroup on each of the spaces $X_{\alpha}$ $(\alpha \in \mathbb{R})$, a fact that we would be hard pushed to prove otherwise.

The final question that arises is to decide to what extent the sum of squares property of $L$ and $M$ is essential for the above proposition and to what extend it holds for more general operators of the form (3.6) or (3.7). This issue I will analyse in a second instalment of this paper. Let me however say that the above proof will give us for free, so to speak, a slightly larger class of operators $L$. We shall explain in Section 9 how we can generalize all the previous machinery to make it work for perturbations of «sums of squares» of the form:

$$
\tilde{L}=\sum_{j} X_{j}^{*} X_{j}+\Lambda^{\alpha}=L+\Lambda^{\alpha}
$$

where $X_{j}$ are $C^{\infty}$ fields as before and $0 \leqslant \alpha \leqslant 2$

$$
\left(\Lambda=(I+\Delta)^{1 / 2}=\left(I-\sum \frac{\partial^{2}}{\partial x_{i}^{2}}\right)^{1 / 2}\right) .
$$

These remarks will be important for our main application in the next section.

\section{Powers of Operators and Subelliptic Distances}

Let $L$ and $M$ be two operators as in Section 7 both of them subelliptic (i.e. $\delta, d<1)$. We shall assume that for two positive $\alpha_{0}, \beta_{0}>0$ we have $\left(X=L^{2}\left(\mathbb{R}^{n}\right)\right)$

$$
\left\|L^{\alpha_{0}} f\right\|_{X} \leqslant C\left\|(I+M)^{\beta_{0}}\right\|_{X}
$$

(it is easy to see that this can only happen if $\alpha_{0} \leqslant \beta_{0}$ ). The issue is to decide to what extent we can conclude from (8.1) that we in fact have

$$
\left\|L^{\nu \alpha_{0}} f\right\|_{X} \leqslant C\left\|(I+M)^{\nu \beta_{0}}\right\|_{X}, \quad \nu>0
$$

or more generally

$$
\left\|L^{\nu \alpha_{0}} f\right\|_{L^{p}} \leqslant C\left\|(I+M)^{\nu \beta_{0}}\right\|_{L^{p}}, \quad \nu>0, \quad 1<p<+\infty .
$$

A few banal remarks are in order. First of all (8.1) implies (8.2) for $0 \leqslant \nu \leqslant 1$ by general operator theory ( $c f$. [23]). Secondly, if (8.2) holds for arbitrary $\nu>0$ then (8.3) also holds for arbitrary $\nu>0,1<p<+\infty$. This is because the operator $L^{z \alpha_{0}}(1+M)^{-z \beta_{0}}$ is $L^{p} \rightarrow L^{p}$ bounded for $\operatorname{Re} z=0$ and if it also is $L^{2} \rightarrow L^{2}$ bounded for $\operatorname{Re} z=\nu>0$ arbitrary we can interpolate.

Let us denote now by

$$
\Lambda_{L}^{\alpha}=\left\{f \in L^{\infty}:|f(x)-f(y)| \leqslant C d_{L}^{\alpha}(x, y)\right\}
$$


for every $0<\alpha \leqslant 1$ where $d_{L}(\bullet, \bullet)$ is the distance induced by the subelliptic operators $L$ ( $c f$. Section 0 ), we also have the analogous definition $\Lambda_{M}^{\alpha}$ of $M$. The validity of (8.3) for all $1<p<+\infty$ and all $\nu \leqslant \nu_{0}$ for some fixed $\nu_{0}$ has the following important consequence

$$
\Lambda_{M}^{\beta} \subsetneq \Lambda_{L}^{\alpha-\epsilon}, \quad \epsilon>0
$$

which in turn (trivialy) implies the following Hölder estimate of one distance with respect to the other

$$
d_{M}^{\beta}(x, y) \leqslant C_{\epsilon} d_{L}^{\alpha-\epsilon}(x, y), \quad \epsilon>0
$$

( $C_{\epsilon}$ does not depend on $x, y$ but, a priori at least, does depend on $\epsilon>0$ ). To see how (8.4) is derived we shall have to use the «Besov spaces» $\Lambda_{p, q}^{\alpha}$ introduced in [15]. To make the distinction between the two operators $L$ and $M$, I shall note by $\Lambda_{p, q}^{\alpha}(L)$ and $\Lambda_{p, q}^{\beta}(M)$ the corresponding spaces for $L$ and $M$.

By standard arguments involving real interpolation (cf. [4], p. 153 where it is easy to check that (4) is also valid in our setting) we see that the hypothesis we have made implies

$$
\Lambda_{p, \infty}^{\beta}(M) \subsetneq \Lambda_{p, \infty}^{\alpha}(L), \quad 1<p<+\infty, \quad \frac{\alpha}{\beta}=\frac{\alpha_{0}}{\beta_{0}}
$$

provided that $\beta<\nu_{0} \beta_{0}$. On the other hand, we always have

$$
\Lambda_{L}^{\alpha} \subsetneq \Lambda_{\infty, \infty}^{\alpha}(L) \subsetneq \Lambda_{p, \infty}^{\alpha}(L) .
$$

What is less standard and very much depends on the subellipticity of $M$ is that for all $\beta>\epsilon>0$ there exists $p=p_{\epsilon}$ large enough such that

$$
\Lambda_{p, \infty}^{\beta}(M) \subsetneq \Lambda_{\infty, \infty}^{\beta-\epsilon}(M) \subsetneq \Lambda_{M}^{\beta-\epsilon} .
$$

To prove the first inclusion of (8.6) one should combine the abstract «dimensional» result of [2] that give these types of inclusions for general «semigroups of finite dimension» together with the rather obvious fact (cf. [12]) that the semigroup $e^{-t M}$ is «finite dimensional» in the sense of [2] provided that $M$ is subelliptic. In other words we have

$$
\left\|e^{-t M}\right\|_{L^{1 \rightarrow L^{\infty}}}=O\left(t^{-m}\right), \quad t \rightarrow 0
$$

for some finite $m>0$.

In (8.5) and (8.6) we also use twice the fact that $\Lambda_{\infty, \infty}^{\alpha}(M)=\Lambda_{M}^{\alpha}(0<\alpha<1)$ this depends on subellipticity and is proved in a manner entirely analogous to the one used for the corresponding classical spaces $\Lambda_{\infty, \infty}^{\alpha}=\Lambda_{\alpha}$ on $\mathbb{R}^{n}$ (cf. [24]). Some of the ideas of [15] have to be used in this proof but the problem is much easier than the one treated in [15]. At any rate, I shall not give the details of 
these proofs at this point because they are totally alien with the spirit of this paper.

The above remarks put together complete the proofs of the required inclusions (8.5), (8.6).

At this stage I shall recall that conversely $d_{M}^{\beta_{0}} \leqslant C d_{L}^{\alpha_{0}}$ implies the estimate (8.1) provided that $0<\alpha_{0}, \beta_{0}<1 / 2$ (cf. [15]). All in all we finally see that to close this circle of ideas and obtain a proof of the theorem announced in the Introduction it suffices to find a way to prove that implication (8.1) implies (8.2).

The implication (8.1) implies (8.2) is an immediate consequence of the considerations of [3] as soon as we know that the semigroup $e^{-t M}$ acts continuously on each Sobolev space $X_{\alpha}$ of the operator $L$. In other words, we are in the situation of Section 7. We can therefore assert straight away that the theorem holds under the subellipticity condition $d+\delta \leqslant 1$ for the two operators $L$ and $M$. It holds in particular if $0 \leqslant d, \delta \leqslant 1 / 2$.

We shall finally show how (8.1) implies (8.2) under the weaker condition $0 \leqslant d \leqslant 1 / 2$ and in fact under a slightly more general condition that depends on $\alpha_{0}, \beta_{0}$. Towards that we shall assume as we may that (8.1) holds for $\alpha_{0}$, $\beta_{0}$ small $\ll 1$ and let us consider the perturbed operator: $\tilde{L}=L+\Lambda^{\gamma}$ where $\gamma=\min \left\{2, \beta_{0} / \alpha_{0}\right\}$ it follows that

$$
\left\|L^{1 / 2} f\right\|_{X}=(L f, f) \leqslant\left\|\tilde{L}^{1 / 2} f\right\|_{X}=(L f, f)+\|f\|_{\gamma / 2} .
$$

But since the semigroup $e^{-t \tilde{L}}$ generated by $\tilde{L}$ acts on the Sobolev spaces $X_{\alpha}$ of $L$. This follows from the fact that $\Lambda^{2}$ is semibounded (in the sense of (7.8)) on $X_{\alpha}$ which makes $\Lambda^{\gamma} 0<\gamma \leqslant 2$ automatically semibounded (cf. end of Section 10) we deduce that

$$
\left\|L^{\nu \alpha_{0}} f\right\|_{X} \leqslant C\left\|\tilde{L^{\nu \alpha_{0}}}\right\|_{X}, \quad \nu>0 .
$$

The same semiboundedness result implies that

$$
L \ll C\left(L^{\alpha_{0}}+\Lambda^{\beta_{0}}\right)^{1 / \alpha_{0}}, \quad \Lambda^{\gamma} \ll C\left(L^{\alpha_{0}}+\Lambda^{\beta_{0}}\right)^{1 / \alpha_{0}}
$$

since we clearly have $L^{\alpha_{0}} \ll L^{\alpha_{0}}+\Lambda^{\beta_{0}}$ and $\Lambda^{\beta_{0}} \ll L^{\alpha_{0}}+\Lambda^{\beta_{0}}$, and since $L$ is also semibounded on $H_{\alpha}$ for the natural scalar product ( $c f$. [3]). (Here $\ll$ indicates the partial order of positive Hilbert space operators: $P \gg 0$ if and only if $(P f, f) \geqslant 0$.) By adding we obtain that

$$
\left(L+\Lambda^{\gamma}\right) \ll C\left(L^{\alpha_{0}}+\Lambda^{\beta_{0}}\right)^{1 / \alpha_{0}}
$$

and since $1 / \alpha_{0}>1$ by abstract operator theory we deduce that

$$
\tilde{L}^{\alpha_{0}}=\left(L+\Lambda^{\gamma}\right)^{\alpha_{0}} \ll C\left(L^{\alpha_{0}}+\Lambda^{\beta_{0}}\right) \ll C M^{\beta_{0}} .
$$


(Since $\Lambda \ll M$.) It remains for us to show that (8.9) implies that

$$
\left\|\tilde{L^{\nu \alpha_{0}}} f\right\|_{X} \leqslant C\left\|M^{\nu \beta_{0}} f\right\|_{X}, \quad \nu>0
$$

and then putting together (8.7) and (8.10) we have a proof of the implication (8.1) implies (8.2).

The estimate (8.10) will follow from (8.9) (cf. [3]) as soon as we know that $d$ the subellipticity index of $M$ and $1-\gamma / 2$ [a quantity that is (if anything) bigger than the subellipticity index of $\tilde{L}$ ] add up to $\leqslant 1$ i.e. it is enough to have

$$
d \leqslant \frac{\beta_{0}}{2 \alpha_{0}}
$$

for the implication (8.1) implies (8.2) to work with this mehod. By the fact that $\beta_{0} \geqslant \alpha_{0}$ it follows in particular that this is always the case when $d \leqslant 1 / 2$ and the proof of our theorem in the Introduction has been completed.

\section{The Perturbed Operator}

Let us consider the operator $\tilde{L}=L+\Lambda^{2 \alpha_{0}}$ of (7.9) $0<\alpha_{0} \leqslant 1$ and let us define the corresponding Sobolev spaces $X_{\alpha}=\left\{f: \tilde{L^{\alpha / 2}} f \in X\right\}$ we shall still denote by $0 \leqslant \delta<1$ the subellipticity index of $\tilde{L}$ i.e. $\|f\|_{1-\delta} \leqslant C\left\|\tilde{L}^{1 / 2}\right\|_{X}$ and we shall also consider a second operator $M$ as in Section 7 with subellipticity index $d<1$. We shall make one more additional hypothesis: We shall assume that $L$ is already subelliptic (but with no control of its subellipticity index) i.e. we shall assume that some finite number of brackets of the fields $X_{j}$ of $L$ span at every point the tangent space. What we shall show is that the proposition of Section 8 still holds and that $e^{-t M}$ acts on each $X_{\alpha}$ space provided that $\delta+d \leqslant 1$.

The proof of that fact relies on one simple observation: The operator $\tilde{L}$ can be written as follows

$$
\tilde{L}=\sum_{j=1}^{n} Y_{j}^{*} Y_{j}
$$

where $Y_{j} \in S_{1,0}^{1}$ and are either one of the fields $X_{j}$ of $L$ or $Y_{j}=\Lambda^{\alpha_{0}}$. With this decomposition we see that all our theory of Sections 4, 5 and 6 (and therefore also Section 7 which gives the required result) goes through, provided that we can prove the equivalent of the singular integral estimate (4.2) in Section 4. Namely all we need to prove is the following estimate

$$
\left\|Y_{i_{1}} Y_{i_{2}} \ldots Y_{i_{k}} f\right\|_{X} \leqslant C\left\|\tilde{L}^{k / 2} f\right\|_{X} .
$$


To show (9.1), by induction on $k$, I shall generalize the problem slightly and assume that the $Y$ 's above consist of exactly $p-X_{i}$ fields and $q-S_{1,0}^{\alpha_{0}}$ operators (with $p+q=k$ ). I shall commute the $Y^{\prime}$ 's in (9.1) between themselves to bring all the $X_{i}$ fields at the beginning (never commute two fields $X_{i}$ between themselves). We obtain thus that the left hand side of (9.1) is

$$
\left\|X_{i_{1}} X_{i_{2}} \ldots X_{i_{p}} S^{q \alpha_{0}} f\right\|_{X}+\cdots
$$

where the ... come from the successive commutators and are identical expressions with a lower $k$. The ... can therefore be dealt with by the induction hypothesis. As for the principal term in (9.2) by our singular integral theorem it can be estimated by $\left\|L^{p / 2} S^{q \alpha_{0}}\right\|_{X}$. This gives the required inductive step by Section 6 since ( $L$ is really $I+L$ )

$$
\left\|L^{p / 2} f\right\|_{H_{q \alpha_{0}}} \approx\left\|L^{p / 2} \Lambda^{q \alpha_{0}} f\right\|_{X} \leqslant C\left\|\left(L+\Lambda^{2 \alpha_{0}}\right)^{(p+q) / 2} f\right\|_{X} .
$$

To see how the last estimate is obtained in (9.3) observe first that by the considerations that lead to (8.8) we have

$$
\begin{aligned}
\left\|L^{p} \Lambda^{s} f\right\| & \leqslant C\left\|\left(L+\Lambda^{r}\right)^{p} \Lambda^{s} f\right\|, \\
\left\|\Lambda^{\alpha_{0} q}\left(L+\Lambda^{t}\right)^{s} f\right\| & \leqslant C\left\|\left(L+\Lambda^{2 \alpha_{0}}\right)^{q / 2}\left(L+\Lambda^{t}\right)^{s} f\right\|, \quad f \in C
\end{aligned}
$$

for $s, t \in \mathbb{R}, p, q \geqslant 0,0 \leqslant r \leqslant 2,0 \leqslant \alpha_{0} \leqslant 1$. It suffices therefore to prove that

$$
\left\|L^{p} \Lambda^{s} f\right\| \leqslant C\left\|\Lambda^{s}\left(L+\Lambda^{r}\right)^{p} f\right\| ; \quad p \geqslant 0, \quad 0 \leqslant r \leqslant 2, \quad s \in \mathbb{R} .
$$

The estimate (9.5) for $p=1$ followes from (9.4) and the commutator estimate ( $c f$. Section 6)

$$
\left\|\left[\Lambda^{s}, L\right] f\right\| \leqslant C\left\|L^{1 / 2} \Lambda^{s} f\right\| \leqslant C\left\|L \Lambda^{s} f\right\|^{1 / 2}\left\|\Lambda^{s} f\right\|^{1 / 2}, \quad f \in C_{0}^{\infty} .
$$

Indeed the right hand side of (9.6) can be estimate (Peter-Paul!) by $\epsilon\left\|L \Lambda^{s} f\right\|+$ $(C / \epsilon)\left\|\Lambda^{s} f\right\|$ and $\epsilon\left\|L \Lambda^{s} f\right\|$ can be absorbed with the left hand side of (9.5) (for $p=1$ ). This same type of argument gives $(9.5)$, by induction, for $p=1,2, \ldots$. Indeed for $p=1,2, \ldots$ we have (use (8.8) again)

$$
\begin{aligned}
\left\|L^{p+1} \Lambda^{s} f\right\| & \leqslant C\left\|\left(L+\Lambda^{r}\right) L^{p} \Lambda^{s} f\right\| \\
& \leqslant C\left\|L^{p} \Lambda^{s}\left(L+\Lambda^{r}\right) f\right\|+C\left\|\left[L, L^{p} \Lambda^{s}\right] f\right\|+C\left\|\left[\Lambda^{r}, L^{p} \Lambda^{s}\right] f\right\| \\
& =I_{1}+I_{2}+I_{3}, \quad f \in C_{0}^{\infty} .
\end{aligned}
$$

$I_{1}$ is estimated by the inductive step (set $\left.g=\left(L+\Lambda^{r}\right) f\right)$. By Section 6 , we have

$$
I_{2} \leqslant C\left\|L^{p+1 / 2} \Lambda^{s} f\right\| \leqslant \epsilon\left\|L^{p+1} \Lambda^{s} f\right\|+C(\epsilon)\left\|\Lambda^{s} f\right\|, \quad \epsilon>0,
$$

and we finish as before by Peter-Paul. Finally we have

$$
I_{3} \leqslant C\left\|L^{p-1 / 2} \Lambda^{r+s} f\right\| \leqslant C\left\|L^{p} \Lambda^{r+s} f\right\| \leqslant C\left\|\Lambda^{r+s}\left(L+\Lambda^{r}\right)^{p} f\right\|=C\left\|\Lambda^{r+s} g\right\|,
$$


$f \in C_{0}^{\infty}, \quad g=\left(L+\Lambda^{r}\right)^{p} f$, by the inductive hypothesis. But

$$
\left\|\Lambda^{r+s} g\right\| \leqslant\left\|\Lambda^{s}\left(L+\Lambda^{r}\right) g\right\|+\left\|\Lambda^{s} L g\right\|
$$

and the case $p=1$ applied to $g$ finishes the proof of (9.5) for $p$ and arbitrary non-negative integer.

The final problem that we have to face is that for (9.3) we need (9.5) for $p=$ half integers (and I am indebted to the referee who pointed out that difficulty to me). In fact as we have said (9.5) holds for arbitrary $p \geqslant 0$. To see how this is done let us use the notation at the end of Section 6 and write (9.5) for fixed $s \in \mathbb{R}$

$$
\|f\|_{Z_{s}^{p}} \leqslant\left\|\left(L+\Lambda^{r}\right)^{z} f\right\|_{Z_{s}^{0}}, \quad f \in C_{0}^{\infty}, \quad z=p .
$$

It is clear that (for fixed $s$ ) $Z_{s}^{p}=Z^{p}$ is a «complex interpolation scale». If we can complexify $z=p+i s$ in (9.7) we will then use the analytic family of operators $\phi(z)=\left(L+\Lambda^{r}\right)^{-z}$ and we are though. That complexification $p \rightarrow p+i s$ amounts simply to showing that $\tilde{L}^{i s}=\left(L+\Lambda^{r}\right)^{i s}=H_{s} \rightarrow H_{s}$ is bounded $(0 \leqslant r \leqslant 2)$. The proof of that fact given in Section 3 , for the operator $L$, also works for the operator $\tilde{L}$ (indeed $\tilde{L} \in S_{1,0}^{2}$ and already in [3] it was shown that $e^{-t \tilde{L}}$ acts on $H_{\alpha}$ ) and we are done.

There is another way (more elegant but also more subtle) to deduce (9.7) for all $z \in \mathbb{C}, \operatorname{Re} z>0$ from the validity of the same inequality for just one value $z=p_{0}>0$. It suffices to apply the theory of [3] on the interpolation scale $Z_{s}^{p}\left(p>0, s\right.$ is fixed. The fact that this scale is not self-dual i.e. $\left(Z_{s}^{p}\right)^{*}$ $=Z_{-s}^{-p}$ (and not $Z_{s}^{-p}$ ) cause no problems). For that we must show that $e^{-t L}$ acts on spaces $Z_{s}^{p}, p=0,2, \ldots$. By a moments reflexion we see that this is a consequence of Section 10 and of the fact that $c_{\lambda}(L)$ is semibounded (in the sense (7.8)) on the spaces $X_{\alpha}(\alpha=0,2,4, \ldots)$ of Section 1. This last fact will be left as an exercise for the (patient) reader. (Hint: $c_{\lambda}(L)=L+X_{j} E$ $+E$ is a «small» perturbation of $L$.) The proof of (9.3) is complete.

\section{The Semiboundedness Property of $\Delta$}

Let $L$ be as before [cf. (7.1)] and let us denote by

$$
\langle f, f\rangle_{n}=\sum_{j=0}^{n} c_{j}\left\langle(1+L)^{2 j} f, f\right\rangle
$$

for $c_{j} \geqslant 0,\left(c_{n}>0\right)$ where $\langle\cdot, \cdot\rangle$ indicates the canonical scalar product on $X=L^{2}$. Thus for any choice of the $c_{j}$ 's is a Hilbert space scalar product on $X_{2 n}, n=0,1, \ldots$ I shall show that (with appropriate choices of the $c_{j}$ 's) the operator $\Delta$ is semibounded in the sence of (7.8) on these scalar products. More 


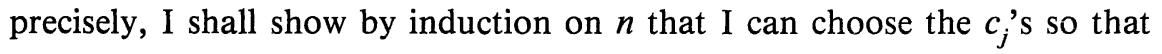

$$
\operatorname{Re}\left\langle\left(\Delta+\lambda_{0}\right) f, f\right\rangle_{n} \geqslant k\left\|\Lambda(I+L)^{n} f\right\|_{X}^{2}, \quad f \in C_{0}^{\infty}
$$

for some $k=k_{n}>0$ and all $\lambda_{0}>0$ large enough.

Assume that the result holds for some $n=0,1, \ldots$. We have then

$$
\left\langle\left(\Delta+\lambda_{0}\right) f, f\right\rangle_{n+1}=\left\langle(I+L)^{2}\left(\Delta+\lambda_{0}\right) f, f\right\rangle_{n}+c_{0}\left\langle\left(\Delta+\lambda_{0}\right) f, f\right\rangle
$$

$\left\langle(I+L)^{2}\left(\Delta+\lambda_{0}\right) f, f\right\rangle_{n}=\left\langle\left(\Delta+\lambda_{0}\right)(I+L) f,(I+L) f\right\rangle_{n}+\langle[\Delta, L] f,(1+L) f\rangle_{n}$.

The first term on the right hand side of (10.1) has a real part that is, by our inductive hypothesis, $\geqslant k_{n}\left\|\Lambda(1+L)^{n+1}\right\|_{X}^{2}$. The second term in the right hand side of (10.1) can be estimated in modulus by

$$
\sum_{j=0}^{n}\left|\left\langle(1+L)^{j}[\Delta, L] f,(I+L)^{j+1} f\right\rangle\right|=\sum\left|\left\langle X_{1} X_{2} \ldots X_{\alpha} \Lambda^{2} E f, Y_{1} \ldots Y_{\beta} f\right\rangle\right|
$$

where $E \in S_{1,0}^{0}, \alpha \leqslant 2 j+1, \beta \leqslant 2 j+2$ and the $X^{\prime}$ 's and $Y$ 's are chosen among the $C_{0}^{\infty}$ fields that define $L\left(=\sum X^{*} X\right)$ as in (7.1). By commutating and taking adjoints we can therefore rewrite every $\langle\cdot, \cdot \bullet\rangle$ on the right as

$$
\begin{aligned}
\left|\left\langle X_{1} X_{2} \ldots X_{\alpha} \Lambda E f, Y_{1}, \ldots, Y_{\beta} \Lambda E f\right\rangle\right| & \leqslant\left\|X_{1}, \ldots, X_{\alpha} \Lambda E f\right\|_{X}\left\|Y_{1}, \ldots, Y_{\beta} \Lambda E f\right\|_{X} \\
& \leqslant C\left\|(I+L)^{\alpha / 2} \Lambda E f\right\|_{X}\left\|(1+L)^{\beta / 2} \Lambda E f\right\|_{X} \\
& \leqslant C\left\|(I+L)^{j+1 / 2} \Lambda E f\right\|_{X}\left\|(1+L)^{j+1} \Lambda E f\right\|_{X} \\
& \leqslant C\left\|(I+L)^{j+1 / 2} \Lambda f\right\|_{X}\left\|(1+L)^{j+1} \Lambda f\right\|_{X} .
\end{aligned}
$$

By the singular integral estimate (4.2) and the estimates that we developed in Sections 4,5 and 6 . But by the log-convexity properties of $\left\|(1+L)^{\nu} \Lambda f\right\|_{X}$ (as a function of $\nu$, since $X=L^{2}$ ) we can finally estimate this scalar product by

$$
\begin{aligned}
& \leqslant\left\|(I+L)^{j+1} \Lambda^{1 / 2} f\right\|_{X}\left[\epsilon\left\|(1+L)^{(j+1)} \Lambda f\right\|_{X}+C(\epsilon)\|\Lambda f\|_{X}\right] \\
& \leqslant \epsilon\left\|(I+L)^{j+1} \Lambda f\right\|_{X}^{2}+C_{1}(\epsilon)\|\Lambda f\|_{X}
\end{aligned}
$$

for arbitrary $\epsilon>0$ and $C(\epsilon), C(\epsilon)>0$. If we put everything together and choose the $c_{0}$ on the last term on the right of (10.1) sufficiently large we obtain the required inductive step.

For odd integers (and other values of $\alpha$ ) we can also show directly that $\Delta$ is a semibounded operator for an appropriate scalar product of $X_{\alpha}$. It is preferable however to proceed indirectly. Observe that $A$ semibounded on some Hilbert space $X$ if and only if

$$
\left\|e^{-t A}\right\|_{X \rightarrow X} \leqslant e^{\lambda_{0} t}
$$

for some $\lambda_{0}>0$ and this estimate, once established for the Hilbert spaces $X_{2 n}$, goes throught to all the other $X_{\alpha}(\alpha \in \mathbb{R})$ by duality and interpolation. It is also 
this estimate that, by subordination ( $c f$. [6]), easily gives the corresponding result for all $A^{\alpha}, 0 \leqslant \alpha \leqslant 1$.

The above proof that I gave is very much in the spirit of this paper but it is very unsatisfactory because it uses the subellipticity of $L$ in a place that it probably has nothing to do. The result is probably correct without that hypothesis. I shall give a proof below of that fact for $\alpha=1$ or 2 .

If $L=\sum X_{j}^{*} X_{j}$, for $\alpha=1$, we much show that

$$
\operatorname{Re}\left(X_{k} \Delta f, X_{k} f\right)+c(\Delta f, f)+c(f, f) \geqslant 0
$$

for appropriate $c>0$. But this is clear enough since $X_{k}^{*}\left[\Delta X_{k}\right] \in S_{1,0}^{2} \bmod$ (skew adjoint terms), which allows us to write

$$
\operatorname{Re}\left(X_{k} \Delta f, X_{k} f\right)=\sum\left\|\Delta^{1 / 2} X_{k} f\right\|+\left(S^{2} f, f\right)
$$

for some $S^{2} \in O P S_{1,0}^{2}$. For $\alpha=2$ we must show that

$$
\operatorname{Re}(L \Delta f, L f)+c_{1} \sum \operatorname{Re}\left(X_{k} \Delta f, X_{k} f\right)+c_{2}(\Delta f, f)+c_{3}(f, f) \geqslant 0
$$

again for appropriate $c$ 's that can be chosen at will. From the case $\alpha=1$ we conclude therefore that it suffices to choose $c_{i}>0$ below so that

$$
\begin{gathered}
\operatorname{Re}(L \Delta f, L f)+c_{1} \sum\left\|\Delta^{1 / 2} X_{k} f\right\|_{X}^{2}+c_{2}\left\|\Delta^{1 / 2} f\right\|_{X}^{2}+c_{3}\|f\|_{X}^{2} \geqslant 0, \\
\operatorname{Re}(L \Delta f, L f)=\left\|\Delta^{1 / 2} L f\right\|_{2}^{2}+\operatorname{Re}([L, \Delta] f, L f),
\end{gathered}
$$

and after a number of easy calculations we finally see that it all amounts to controlling from below the expression

$$
\operatorname{Re} \sum_{i, j}\left(A_{i j} X_{i} f, X_{j} f\right)
$$

where $A_{i j}=X_{j}\left[\Delta, X_{i}\right]$. This however is easy for if we denote by $Q=\left(A_{i j}\right)_{i, j=1}^{n}$ then it is clear that

$$
Q+Q^{*}=2\left(\left[\left[\Delta X_{i}\right] X_{j}\right]\right)_{i, j} \quad \bmod \left(O P S_{1,0}^{2}\right)
$$

and the control from below is thus supplied by the second term of (10.2).

\section{Final Remarks on the Method of the Proofs}

The main functional analytic tool used to obtain the critical indices in Sections 4,5 and 6 was the machinery of real interpolation. It turns out that there is another approach, in some ways dual to the one used, which could have been adopted instead. This approach is interesting in its own right and without going into details I will describe its main features. 
The key is to prove that

$$
\left\|\left(c_{\lambda}-c_{\mu}\right)\left(A^{\sigma} e^{-t A}\right)\right\|_{\alpha \rightarrow \alpha}=O\left(t^{-\operatorname{Re} \sigma+1 / 2}\right)
$$

for $\lambda, \mu, \alpha \in \mathbb{R}$ and $\operatorname{Re} \sigma>1 / 2 .\|\cdot\|_{\alpha \rightarrow \beta}$ refers here and in what follows to the $H_{\alpha} \rightarrow H_{\beta}$ norm. It is clear by the observation that $\left\|c_{\lambda}(\cdot)\right\|_{\alpha \rightarrow \alpha}=\left\|c_{\lambda+\alpha}(\cdot)\right\|_{0 \rightarrow 0}$ and by taking differences that we can assume that $\alpha=0$ (and even $\lambda=0$ ). It is also enough to assume that $\sigma=1,2, \ldots$ and then use the standard formulae for the powers of an operator with positive real part.

The problem thus becomes to establish

$$
\left\|\left(I-c_{\lambda}\right)\left(\frac{d^{k}}{d t^{k}} e^{-t A}\right)\right\|_{X \rightarrow X}=O\left(t^{1 / 2-k}\right)
$$

for $k=1,2, \ldots$. There seems to be no automatic way of obtaining this without introducing some new idea. The idea is to consider the holomorphic function

$$
\Phi(z)=\left(1-c_{\lambda}\right)\left(e^{-z A}\right), \quad|\operatorname{Arg} z|<\pi / 4
$$

and to start by establishing that $\|\Phi(z)\|_{X \rightarrow X}$ (or even $\|\cdot\|_{\alpha \rightarrow \alpha}$ ) is $O\left(|z|^{1 / 2}\right.$ ) in that sector. This is done just as in Section 5 since $e^{-z A}$ is a holomorphic semigroup. Then we use Cauchy's theorem and the required estimate for $\left(d^{k} / d z^{k}\right) \Phi(z)$ is obtained.

Having estimated (11.1) we then proceed to estimate

$$
\left\|A^{\sigma}\left(c_{\lambda}-c_{\mu}\right)\left(e^{-t A}\right)\right\|_{\alpha \rightarrow \alpha}=O\left(t^{-\operatorname{Re} \sigma+1 / 2}\right) .
$$

This time the result clearly also holds for $\operatorname{Re} \sigma=0$ and therefore for any $\operatorname{Re} \sigma \geqslant 0$. This for $\sigma=1,2, \ldots$ is deduced from (11.1) and the formula

$$
\left(I-c_{\mu}\right)\left(A^{k} e^{-t A}\right)=A^{k}\left[\left(I-c_{\mu}\right) e^{-t A}\right]+\left[\left(I-c_{\mu}\right)\left(A^{k}\right)\right] c_{\mu}\left(e^{-t A}\right)
$$

where (for $k=1,2, \ldots)\left(1-c_{\mu}\right)\left(A^{k}\right)$ is dealt with by pseudo-differential calculus as before. To obtain (11.2) we finally have to make a rather subtle use of complex interpolation. Observe that $\left[E, A^{\sigma} e^{-t A}\right]$ and $A^{\sigma}\left[E e^{-t A}\right]$ are obtained analogously and they are in fact simpler since the last step that uses complex interpolation is not needed. I will not give the details.

A final step in this approach to our theory consists in proving estimates of the type

$$
\left\|\left(1-c_{\lambda}\right)\left(X_{1} \cdots X_{k} e^{-t A}\right)\right\|_{X \rightarrow X}=O\left(t^{(1-k) / 2}\right)
$$

for $A$-sub-unit fields $X_{j}$. This, in term, allows us to show at once that the multiple Riesz transforms $R=X_{1} \cdots X_{k} A^{-k / 2}$ is an $H_{\alpha} \rightarrow H_{\alpha}$ bounded 
operator. To prove the estimate (11.4) we first reduce it to the corresponding estimate of $X_{1} \cdots X_{k}\left(I-c_{\lambda}\right)\left(e^{-t A}\right)$ by the analogue of formula (11.3) and then we use the singular integral theorem (4.2) to reduce it to (11.2). The Riesz transforms then follow from (11.4) by the same «trick» that was used in Section 3 for the pure imaginary powers ( $c f$. Section 3). I will not give the details here.

\section{An Example}

Some of the results of this paper will be illustrated by the following example

$$
\begin{gathered}
L_{i}=X^{*} X+Y^{*} Y+Z_{i}^{*} Z_{i}, \quad i=1,2 \\
X=\frac{\partial}{\partial x}, \quad Y=x \frac{\partial}{\partial y}, \quad Z_{i}=a_{i}(y) e^{-1 / y^{2}} \frac{\partial}{\partial y}, \quad(x, y \in \mathbb{R})
\end{gathered}
$$

where the $0<a_{i}(y) \in C^{\infty}(\mathbb{R} \backslash\{0\})$ are chosen to be, together with all their derivatives, $O\left(|y|^{-n}\right)$ as $y \rightarrow 0$ so that the two above operators are $C^{\infty}$ subelliptic Hörmander operators in $\mathbb{R}^{2}$ as in Section 7 with $\delta=d=1 / 2$.

Then $a_{i}, i=1,2$ will be chosen so that

$$
a_{1} / a_{2} \notin L^{\infty}(\mathbb{R}), \quad a_{1} / a_{2} \in L_{\mathrm{loc}}^{p}(\mathbb{R}) ; \quad \text { for every } \quad 1<p<+\infty
$$

(e.g. $a_{1}(y)=-\log |y|, a_{2}(y) \equiv 1$ for small $\left.y\right)$.

From this it follows that for all $C>0$ we have

$$
\left\|L_{1}^{1 / 2} f\right\|_{2} \nless C\left[\left\|L_{2}^{1 / 2} f\right\|_{2}+\|f\|_{2}\right], \quad f \in C_{0}^{\infty} .
$$

Here we should note that $\left\|L_{i}^{1 / 2} f\right\|_{2}=\left\|\nabla_{i} f\right\|_{2}$ with

$$
\nabla_{i} f(x)=\left(\left|\frac{\partial f}{\partial x}\right|^{2}+\left(x^{2}+a_{i}^{2}(y) e^{-2 / y^{2}}\right)\left|\frac{\partial f}{\partial y}\right|^{2}\right)^{1 / 2} .
$$

It is an automatic consequence of (12.2) (or it can be seen by direct calculation) that the corresponding distances satisfy

$$
d_{2}(\cdot, \cdot) \nless C d_{1}(\cdot, \cdot) ; \text { for every } C>0 .
$$

We shall see however that for $\Omega \subset \subset \mathbb{R}^{2}$ and for all $\epsilon>0$ there exists $C>0$ such that

(12.3) $d_{2}(\cdot, \cdot) \leqslant C_{\epsilon} d_{1}^{1-\epsilon}(\cdot, \cdot),\left\|L_{1}^{1 / 2-\epsilon} f\right\|_{2} \leqslant C_{\epsilon}\left[\left\|L_{2}^{1 / 2} f\right\|_{2}+\|f\|_{2}\right], f \in C_{0}^{\infty}(\Omega)$.

The above two estimates are in fact equivalent because of our main theorem. To see the second estimate (12.3) observe that the second condition of (12.1) 
and Hölder's inequality implies that for all $\delta>0$ there exists $C_{\delta}>0$ such that

$$
\left\|\nabla_{1} f\right\|_{L^{2-\delta}} \leqslant C_{\delta}\left(\left\|\nabla_{2} f\right\|_{L^{2}}+\|f\|_{L^{2}}\right), \quad f \in C_{0}^{\infty} .
$$

The behaviour at infinity is irrelevant in the above considerations but the reader can easily check, if he so wishes, that (12.4) holds even globally provided that $L_{i}$ are correctly modified at infinity.

At any rate what counts here are the following two estimates

$$
\left\|\nabla_{1} f\right\|_{L^{p}}+\|f\|_{L^{p}} \approx\left\|L_{i}^{1 / 2} f\right\|_{L^{p}}+\|f\|_{L^{p}}, \quad 1<p<+\infty, \quad f \in C_{0}^{\infty} .
$$

And also for every $\epsilon>0$, small enough, there exists $\delta>0$ and $C>0$ such that

$$
\left\|L_{2}^{1 / 2-\epsilon} f\right\|_{L^{2}}+\|f\|_{L^{2}} \leqslant C\left[\left\|L_{2}^{1 / 2} f\right\|_{L^{2-\delta}}+\|f\|_{L^{2-\delta}}\right], \quad f \in C_{0}^{\infty} .
$$

(12.4), (12.5) and (12.6) put together imply (12.3).

The estimate (12.5) is the M. Riesz theorem for subelliptic operators already used in sections 3 and 4, cf. [8], [9]. The estimate (12.6) was already used in a slightly different form in Section 8 and is a consequence of the abstract Hardy-Littlewood theory and the finite dimensionality of the semigroup $e^{-t L_{2}}, c f$. [2], [12].

Acknowledgement. I am very grateful to Antonio Sánchez-Calle who read the manuscript in great detail and who made some very pertinent points.

\section{References}

[1] Butzer, P.L. and Berens, H. Semigroups of Operators and Applications, Springer-Verlag, 1967.

[2] Coulhon, T. and Saloff-Coste, L. Theorem de Sobolev pour les semigroupes d'operateurs et application aux groupes de Lie unimodulaires, C.R. Acad. Sci. Paris 309, 289-294.

[3] Varopoulos, N.Th. Puissances des opérateurs pseudo-différentiels, C.R. Acad. Sci. Paris 310(1990), 769-774.

[4] Bergh, J. and Löfström, J. Interpolation Spaces, an Introduction, SpringerVerlag, 1976.

[5] Hörmander, L. The Analysis of Linear Partial Differential Operators, vol. III, Springer-Verlag, 1980

[6] Yosida, K. Functional Analysis, Springer-Verlag, 1978.

[7] Fefferman, C. and Sánchez-Calle, A. Fundamental Solutions of Second Order Subelliptic Operators, Annals of Math. 124(1986), 247-272.

[8] Sánchez-Calle, A. $L^{p}$ Estimates for Degenerate Elliptic Equations, Revista Mat. Iberoamericana 4(1988), 177-185.

[9] Christ, M. Lectures on Singular Integral Operators, A.M.S. Regional Conf. in Math. 77. 
[10] Jerison, S. and Sánchez-Calle, A. Subelliptic Second Order Differential Operators, Lecture Notes Math. 1277.

[11] Fefferman, C. L. and Phong, D. H. Subelliptic Eigenvalue Problems, Proc. of the Conference on Harmonic Analysis in Honor of A. Zygmund. Wadsworth Math. Series (1981), 590-606.

[12] Varopoulos, N. Th. Opérateurs sous-elliptiques du second ordre, C. R. Acad. Sci. Paris 308(1989), 437-440.

[13] Oleiknic, O. and Radkevitch, E. Second-Order Equations with Non-Negative Characteristic Form, Amer. Math. Soc., Providence, 1973.

[14] Hörmander, L. Hypoelliptic Second Order Differential Equations, Act. Math. 119(1967), 147-171.

[15] Varopoulos, N. Th. Distances associées aux opérateurs sous-elliptiques du second ordre, C. R. Acad. Sci. Paris 309(1989), 663-667.

[16] Beals, R. Characterization of Pseudodifferential Operators and Aplications, Duke Math. J. 44(1977), 45-57.

[17] Beals, R. Weighted Distribution Spaces and Pseudodifferential Operators, Journal d'Analyse Mathematique, 39(131-187), 1981.

[18] Coifman, R. R. and Meyer, Y. Au delà des operateurs pseudo-différentiels, $A s$ terisque, 57(1978), 1-185.

[19] Beals, R. $\quad L^{p}$ and Hölder Estimates for Pseudodifferential Operators, Ann. Inst. Fourier 29(1979), 239-260.

[20] Stein, E. M. Topics in Harmonic Analysis Related to the Littlewood-Paley Theory, Princeton University Press, 1970.

[21] Lohoué, N. and Varopoulos, N. Th. Remarques sur les transformées de Riesz sur les groupes de Lie nilpotents, C. R. Acad. Sci. Paris 301(1985), 559-560.

[22] Rothschild, L. P. and Stein, E. M. Hypoelliptic Differential Operators and Nilpotent Groups, Acta. Math. 137(1976), 247-320.

[23] Davies, B. M. One-Parameter Semigroups, Academic Press, 1980.

[24] Stein, E. M. Singular Integrals and Differentiability Properties of Functions, Princeton University Press, 1970.

Recibido: 26 de junio de 1991.

N. Th. Varopoulos Departement de Mathematiques

Université de Paris VI

Place Jussieu

Paris 75005

FRANCE 